TRANSACTIONS OF THE

AMERICAN MATHEMATICAL SOCIETY

Volume 364, Number 4, April 2012, Pages 1737-1770

S 0002-9947(2011)05360-2

Article electronically published on November 15, 2011

\title{
GIT STABILITY OF WEIGHTED POINTED CURVES
}

\author{
DAVID SWINARSKI
}

\begin{abstract}
Here we give a direct proof that smooth curves with distinct marked points are asymptotically Hilbert stable with respect to a wide range of parameter spaces and linearizations. This result can be used to construct the coarse moduli space of Deligne-Mumford stable pointed curves $\bar{M}_{g, n}$ and Hassett's moduli spaces of weighted pointed curves $\bar{M}_{g, \mathcal{A}}$. (The full construction of the moduli spaces is not given here, only the stability proof.) This proof follows Gieseker's approach to reduce the GIT problem to a combinatorial problem, although the solution is very different. The action of any 1-PS $\lambda$ on a curve $C \subset \mathbf{P}^{N}$ gives rise to weighted filtrations of $H^{0}\left(C, \mathcal{O}_{C}(1)\right)$ and $H\left(C, \mathcal{O}_{C}(m)\right)$. We give a recipe in terms of the combinatorics of the base loci of the stages of these filtrations for showing that $C$ is stable with respect to $\lambda$.
\end{abstract}

\section{INTRODUCTION}

In the 1960s, Mumford established Chow stability of smooth unpointed genus $g$ curves embedded by complete linear systems of degree $d \geq 3 g$. In the late 1970s, Gieseker established asymptotic Hilbert stability of smooth curves under complete linear systems of degree $d \geq 2 g+1$, and Mumford used Gieseker's ideas to improve his Chow result to $d \geq 2 g+1$. (Here, the word "asymptotic" describes the linearizations under consideration; these will be described in detail in Section 1.1.) Both of them then use an indirect argument to show that nodal Deligne-Mumford stable (DM-stable) curves are GIT-stable. The case of curves with marked points was not solved until 2006, when Elizabeth Baldwin proved that pointed DM-stable curves are asymptotically Hilbert stable. (Actually, she proved GIT-stability for pointed stable maps, which includes pointed stable curves as a special case.) Her argument is a delicate induction: elliptic tails are glued to the marked points one by one, ultimately relating GIT-stability of an $n$-pointed genus $g$ curve to Gieseker's result for genus $g+n$ unpointed curves.

One might seek to improve on these results in several ways. First, one might seek to construct the moduli spaces of stable weighted pointed curves $\bar{M}_{g, \mathcal{A}}$ introduced by Hassett ([Hass $)$. It appears that Baldwin's proof can accommodate some, but not all, sets of weights. Second, one might wish to study quotients for linearizations not covered by Gieseker's (and hence Baldwin's) result. Third, one might wish to study GIT for $\nu$-canonically embedded curves for small $\nu$. The Log Minimal Model Program for $\bar{M}_{g}$, begun by Keel and Hassett and Hyeon in [HH] and continued by many others, has generated interest in these quotients and their pointed analogues. Gieseker's proof works in these degrees for smooth unpointed curves, but due to its

Received by the editors August 25, 2009 and, in revised form, March 26, 2010.

2010 Mathematics Subject Classification. Primary 14L24, 14H10; Secondary 14D22.

Key words and phrases. Hilbert stability.

(C)2011 American Mathematical Society Reverts to public domain 28 years from publication 
use of elliptic tails, Baldwin's proof cannot be used to study the analogous pointed curves, as elliptic tails are known to be GIT-unstable for $\nu \leq 4$.

To construct the moduli spaces or $\bar{M}_{g, n}$ or $\bar{M}_{g, \mathcal{A}}$ via GIT, one must describe parameter spaces $J$ whose points parametrize embedded DM-stable weighted pointed curves $\left(C \subset \mathbf{P}^{N}, P_{1}, \ldots, P_{n}, \mathcal{A}\right)$, and then describe linearizations for which these points coincide with the GIT-(semi)stable locus. For most of the paper, we address a different, but related, problem:

- We ignore the set of weights $\mathcal{A}$ and study embedded pointed curves $(C \subset$ $\left.\mathbf{P}^{N}, P_{1}, \ldots, P_{n}\right)$.

- We assume that the curve $C$ is smooth.

The main result of this paper, Theorem 7.1, is a direct (rather than inductive) proof that smooth curves with marked points are GIT-stable with respect to a wide range of parameter spaces and linearizations. Armed with this result, one may proceed to show that all DM-stable weighted pointed curves are GIT-stable for certain linearizations, as desired, and construct the coarse moduli spaces of DM-stable weighted pointed curves $\bar{M}_{g, n}$ and $\bar{M}_{g, \mathcal{A}}$. (This result is stated without proof as Theorem 8.1.) The proof presented here applies to 2- or 3-canonical linear systems. It is logically independent of Gieseker's, though many of the ideas used are inspired by his work, and in the unpointed case it works for approximately the same linearizations. Our approach follows Gieseker's in reducing the GIT problem to a combinatorial problem, though the solution is very different from his. Here is a description of our approach.

Strategy for the main GIT-stability result. Let $x$ be a point parametrizing an embedded smooth pointed curve $\left(C \subset \mathbf{P}^{N}, P_{1}, \ldots, P_{n}\right)$. We reformulate the numerical criterion in a way that permits a more combinatorial approach. A 1-PS $\lambda$ of $S L(N+1)$ induces a weighted filtration of $H^{0}(C, \mathcal{O}(1))$ and a weighted filtration of $H^{0}\left(C, \mathcal{O}_{C}(m)\right)$. The value of Mumford's function $\mu^{L}(x, \lambda)$ may be interpreted as the "minimum weight of a basis of $H^{0}\left(C, \mathcal{O}_{C}(m)\right)$ compatible with this filtration plus a contribution from the marked points". (From now on, whenever we refer to a basis of $H^{0}\left(C, \mathcal{O}_{C}(m)\right)$, we always implicitly mean one that is compatible with the weighted filtration.) The numerical criterion says that if $\mu^{L}(x, \lambda)$ is sufficiently small, then $x$ is GIT-stable with respect to $\lambda$. Any basis therefore gives an upper bound for $\mu^{L}(x, \lambda)$, so the goal becomes: find a basis of sufficiently small weight.

Our main tool for computing a bound for the weight of a basis is something we call a profile. This is a graph which may be associated to any filtration of a vector space such that the weight decreases at each stage. Suppose $\widetilde{F}_{\bullet}$ is such a filtration of $H^{0}\left(C, \mathcal{O}_{C}(m)\right)$. (Here we use tildes for filtrations of $H^{0}\left(C, \mathcal{O}_{C}(m)\right)$; no tilde indicates a filtration of $H^{0}(C, \mathcal{O}(1))$.) Suppose the weight on the $k^{\text {th }}$ stage of $\widetilde{F}_{\bullet}$ is $\widetilde{r}_{k}$. Then the profile associated to $\widetilde{F}_{\bullet}$ is just the decreasing step function in the first quadrant of the (codimension $\times$ weight)-plane whose value is $\widetilde{r}_{k}$ over the interval [ $\left.\operatorname{codim} \widetilde{F}_{k}, \operatorname{codim} \widetilde{F}_{k+1}\right)$. Given any profile, it is possible to choose a basis whose weight is less than or equal to the area under the profile.

There is a notion of an absolute weight filtration on $H^{0}\left(C, \mathcal{O}_{C}(m)\right)$ (see Section 1.3). The area under its profile is the minimum weight of a basis, and hence computes Mumford's function $\mu^{L}(x, \lambda)$. This is the most natural filtration to consider, but it is too difficult to compute. So, like Gieseker, we study other filtrations. 
The action of a 1-PS $\lambda$ induces a filtration $V \bullet$ of $H^{0}(C, \mathcal{O}(1))$. By considering specific spaces of degree $m$ monomials in elements of $V$ diagonalizing the $\lambda$-action, Gieseker produces a very straightforward filtration $\widetilde{V}_{\bullet}$ of $H^{0}\left(C, \mathcal{O}_{C}(m)\right)$ as well as a second, slightly fancier filtration $\widetilde{G}_{\bullet}$. Gieseker is able to show that the weight (or area) associated to $\widetilde{G} \bullet$ is sufficiently small to establish $\lambda$-stability of smooth unpointed curves. Unfortunately, as we show with a concrete example, the analogue of $\widetilde{G}_{\bullet}$ is not sufficient to establish $\lambda$-stability when there are marked points.

One could try to improve $\widetilde{G}_{\bullet}$, but it is too difficult (at least for us) to show that the sum of its area and the marked points contribution is sufficiently small. Therefore we use $\widetilde{V}_{\bullet}$ as a starting point to build a new filtration, $\widetilde{X}_{\bullet}$, which is obtained by taking spans of carefully chosen spaces of monomials. In a convenient (though not rigorous) soundbite: where Gieseker uses monomials, our proof uses polynomials. The recipe for $\tilde{X}_{\bullet}$ is given in terms of the combinatorics of the base loci of the stages of the filtration $V_{\bullet}$. Although $\tilde{X}_{\bullet}$ is rather tedious to define, it has the virtue that we can bound the sum of its area and the marked points contribution sufficiently well to show that smooth curves with marked points are GIT-stable. The key new ingredients in our proof are the definition/choice of $\widetilde{X}_{\bullet}$, an easy but important lemma (Lemma 3.2) which allows us to compute spans of spaces of monomials in the $V_{j}$ 's using multiplicities of points in the base loci, and the combinatorial argument (see the proof of Lemma 6.1) which allows us to effectively bound the sum of the marked points contribution and the area of the profile associated to $\tilde{X}_{\bullet}$.

Gieseker's proof establishes stability for smooth unpointed curves embedded by complete linear systems of degree $d \geq 2 g+1$. At the present time it is necessary for us to make the hypotheses:

- If $n=0$, the parameter space satisfies $N \geq 2 g-2$, or, equivalently, $d \geq$ $3 g-3$.

- If $n \geq 1$, then either the parameter space satisfies $N \geq 2 g-1$, or the linearization satisfies the following condition (the notation is explained in Section 1.1): $b>\frac{g-1}{N}$.

Outline. In Section 1, we describe the GIT problem carefully, specifying the parameter spaces and linearizations we will consider, and reformulate the numerical criterion in the form in which we shall use it. Profiles are defined here. In Section 2 we review Gieseker's proof, with a few enhancements, to fix notation. In Section 3 we give an example showing why his proof does not suffice for marked points, and a hint illustrating how we will go about fixing it.

Throughout Sections 13 we steadily extract combinatorial data from the algebrogeometric action of a 1-PS $\lambda$ acting on the Hilbert point of a smooth pointed curve. The last result of this type is Lemma 3.2. which allows us to compute codimensions of spans of monomial-type sublinear series of $H^{0}\left(C, \mathcal{O}_{C}(m)\right)$ using only the multiplicities of points in the base loci. After this, the problem becomes almost entirely combinatorial.

In Section 4, we produce the filtration $\widetilde{X}_{\bullet}$ on $H^{0}\left(C, \mathcal{O}_{C}(m)\right)$ which is built using the filtration $\widetilde{V}_{\bullet}$ as scaffolding. The goal is now to show that the area under the profile for $\widetilde{X} \bullet$ plus the contribution from the marked points is less than the bound specified by the numerical criterion. 
This is established in two steps: first, we describe a second, simpler graph called the virtual profile which is bounded above by the profile for $\tilde{X}_{\bullet}$. Up to rounding errors, it is the graph of the piecewise linear function connecting the left endpoints of the steps in the weight profile. The virtual profile is not really the profile of any filtration, nor does it compute or bound the weight of a basis. The most rigorous interpretation we have for it is on the level of graphs. Again, while it is easy to compute the area of the profile, when it is time to add the contribution from the marked points, it is easier to do this with the virtual profile than with the profile. In Section 5 we bound the discrepancy between the areas of the two graphs and show that this is relatively small when $m$ is large. Then in Section 6 we bound the sum of the area under the virtual profile for $\widetilde{X}_{\bullet}$ and the weight from the marked points. Everything comes together in Section 7 to show that smooth pointed curves with marked points have GIT-stable Hilbert points. In Section 8 we describe an application of the GIT-Stability Theorem to constructing moduli spaces.

Here is a picture illustrating the profile and virtual profile associated to an example that is explained in detail in Section 4.2 .

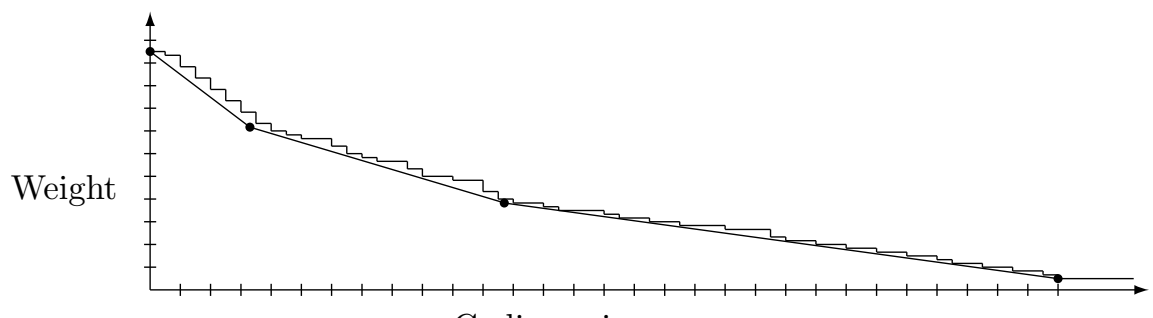

Codimension

FiguRE 1. Example profile and virtual profile

\section{The GIT SETUP FOR POINTED CURVES}

1.1. The parameter spaces and linearizations. We investigate GIT-stability for the following setup. Let $P(t):=d t-g+1$ be a degree one polynomial. We form the incidence locus $I \subset \operatorname{Hilb}\left(\mathbf{P}^{N}, P(t)\right) \times \prod_{i=1}^{n} \mathbf{P}^{N}$, where the points in the projective space factors lie on the curve in $\mathbf{P}^{N}$ parametrized by the point in the first factor. We study the GIT stability of points of $I$.

To construct the moduli spaces $\bar{M}_{g, \mathcal{A}}$ (see the outline in Section 8 ), one would restrict his attention to curves which are embedded by linear series of the form $\left(\omega\left(\sum a_{i} P_{i}\right)\right)^{\nu}$. For that reason, we refer to the weights $\mathcal{A}$ as the embedding weights (as opposed to the linearization weights $\mathcal{B}$ which are defined below). However, we do not need to restrict our attention to linear series of the form $\left|\left(\omega\left(\sum a_{i} P_{i}\right)\right)^{\nu}\right|$ yet; the GIT-Stability Theorem, Theorem 7.1, is more general than that. We do not assume that $C \subset \mathbf{P}^{N}$ is pluricanonically embedded, or even that the degree of $C \subset \mathbf{P}^{N}$ matches the degree of the pluricanonical embedding. Indeed, we will not see the embedding weights $\mathcal{A}$ again until Section 8 . All we need is that the embedding $C \subset \mathbf{P}^{N}$ is by a complete linear system, and some precise degree/dimension bounds in terms of the genus, which will be carefully stated at the end of Theorem 7.1 .

To do GIT, one must specify a linearization on the $G$-space (here, $I$ ). We do this by embedding $\operatorname{Hilb}\left(\mathbf{P}^{N}, P(t)\right) \times \prod_{i=1}^{n} \mathbf{P}^{N}$ equivariantly in a high-dimensional projective space and use its $\mathcal{O}(1)$. 
Let $C \subset \mathbf{P}^{N}$ be a subscheme with Hilbert polynomial $P(t)$. For sufficiently large $m, m_{i}^{\prime}$, the maps

$$
\begin{array}{ll}
\operatorname{ev}_{C}^{m}: & H^{0}\left(\mathbf{P}^{N}, \mathcal{O}_{C}(m)\right) \rightarrow H^{0}\left(C, \mathcal{O}_{C}(m)\right), \\
\operatorname{ev}_{P_{i}}^{m_{i}}: & H^{0}\left(\mathbf{P}^{N}, \mathcal{O}\left(m_{i}^{\prime}\right)\right) \rightarrow H^{0}\left(P_{i}, \mathcal{O}_{P_{i}}\left(m_{i}^{\prime}\right)\right) \cong \mathbb{C}
\end{array}
$$

are surjective. The first map gives rise to an embedding of the Hilbert scheme in a Grassmannian, which in turn embeds in a projective space by the Plücker embedding. The maps in the second line correspond to $m_{i}^{\prime}$-uple embeddings of $\mathbf{P}^{N}$. Finally, a Segre embedding of all these projective spaces yields an equivariant embedding of $\operatorname{Hilb}\left(\mathbf{P}^{N}, P(t)\right) \times \prod_{i=1}^{n} \mathbf{P}^{N}$ into a high-dimensional projective space, as desired.

To specify a linearization on $I \subset \operatorname{Hilb}\left(\mathbf{P}^{N}, P(t)\right) \times \prod_{i=1}^{n} \mathbf{P}^{N}$, it suffices to specify the ratios between $m$ and each $m_{i}^{\prime}$. We will do this as follows: let $\mathcal{B}=\left(b_{1}, \ldots, b_{n}\right) \in$ $\mathbb{Q}^{n} \cap[0,1]^{n}$ be a set of weights, which we call the linearizing weights. Then set $m_{i}^{\prime}=b_{i} m^{2}$. Finally, write $b:=\sum_{i=1}^{n} b_{i}$.

1.2. The numerical criterion for our setup. By being a little more explicit, we obtain a useful reformulation of the numerical criterion.

In Gieseker's paper and in this paper we use Grothendieck's convention that if $V$ is a vector space, then $\mathbf{P}(V)$ is the set of nonzero linear forms on $V$ up to scaling. One consequence of this convention is that the numerical criterion takes the opposite sign from that in GIT.

Let $X$ be a projective algebraic scheme with the action of a group $G$ linearized on a very ample line bundle $L$. Let $\lambda: \mathbb{G}_{m} \rightarrow G$ be a 1-PS of $G$. Choose a basis $\left\{e_{0}, \ldots, e_{N}\right\}$ of $H^{0}(X, L)$ diagonalizing the $\lambda$ action and ordered so that the weights $r_{0} \leq \cdots \leq r_{N}$ increase. The weights on the dual basis then have the opposite signs: $-r_{0}, \ldots,-r_{N}$.

A point $x \in X$ is represented by some nonzero $\hat{x}=\sum_{i=0}^{N} x_{i} e_{i}^{\vee} \in H^{0}(X, L)^{\vee}$. Define

$$
\mu^{L}(x, \lambda):=\min \left\{r_{i} \mid x_{i} \neq 0\right\}
$$

Then, with our sign conventions, we have the following characterization of GITstability:

Theorem 1.1 (cf. [GIT] Theorem 2.1).

$$
\begin{aligned}
x \in X^{s s}(L) & \Longleftrightarrow \mu^{L}(x, \lambda) \leq 0 \text { for all } 1-P S \lambda \neq 0, \\
x \in X^{s}(L) & \Longleftrightarrow \mu^{L}(x, \lambda)<0 \text { for all } 1-P S \lambda \neq 0 .
\end{aligned}
$$

In our situation $X$ is the incidence scheme $I$, the point $x \in X$ parametrizes an embedded pointed curve $\left(C \subset \mathbf{P}^{N}, P_{1}, \ldots, P_{n}\right)$, the scheme $I$ is embedded in $\mathbf{P}\left(\bigwedge^{P(m)} \operatorname{Sym}^{m} V \otimes \bigotimes_{i=1}^{n} \operatorname{Sym}^{m_{i}^{\prime}} V\right)$, where $V=H^{0}\left(\mathbf{P}^{N}, \mathcal{O}_{\mathbf{P}^{N}}(1)\right)$, and $L$ is the $\mathcal{O}(1)$ on this high-dimensional projective space. Let $\lambda$ be a 1-PS of $S L(V)$. One particularly nice basis of $\bigwedge^{P(m)} \mathrm{Sym}^{m} V \otimes \bigotimes_{i=1}^{n} \operatorname{Sym}^{m_{i}^{\prime}} V$ is given by elements of the form

$$
\left(M_{1} \wedge \cdots \wedge M_{P(m)}\right) \otimes\left(M_{1}^{\prime}\right) \otimes \cdots \otimes\left(M_{n}^{\prime}\right)
$$

where each $M_{j}$ is a monomial of degree $m$ and each $M_{i}^{\prime}$ is a monomial of degree $m_{i}^{\prime}$ in the basis elements of $V$ diagonalizing $\lambda$. 
The numerical criterion may be translated as follows: a point of $I$ is stable with respect to $\lambda$ if and only if there is a basis element of the form (1) such that:

(i) the images of the $M_{\ell}$ under the evalution map form a basis of $H^{0}\left(C, \mathcal{O}_{C}(m)\right)$,

(ii) $M_{i}^{\prime}$ does not vanish at $P_{i}$,

(iii) the $S L(N+1)$ weights satisfy

$$
\sum_{\ell=1}^{P(m)} \mathrm{wt}_{\lambda}\left(M_{\ell}\right)+\sum_{i=1}^{n} \mathrm{wt}_{\lambda}\left(M_{i}^{\prime}\right)<0 .
$$

We normalize the $\lambda$ weights so that they decrease to 0 and sum to 1 . If $s_{N}, \ldots, s_{0}$ are the original weights (so $s_{N} \geq \cdots \geq s_{0}$ and $\sum s_{j}=0$ ), then the desired transformation is $r_{j}=\left(s_{N-j}-s_{0}\right) /\left((N+1)\left|s_{0}\right|\right)$. Also, we write

$$
\begin{aligned}
A & :=\sum_{\ell=1}^{P(m)} \mathrm{wt}_{\lambda}\left(M_{\ell}\right), \\
T & :=\sum_{\ell=1}^{P(m)} \mathrm{wt}_{\lambda}\left(M_{\ell}\right)+\sum_{i=1}^{n} \mathrm{wt}_{\lambda}\left(M_{i}^{\prime}\right)
\end{aligned}
$$

for parts of the left hand side of condition (iii) above.

Lemma 1.2. Condition (iii) above with the unnormalized weights $s_{j}$ is equivalent to the following condition:

(iii') With the normalized weights $r_{j}$, the following inequality is satisfied:

$$
\begin{aligned}
T:=\sum_{\ell=1}^{P(m)} \mathrm{wt}_{\lambda}\left(M_{\ell}\right)+\sum_{i=1}^{n} \mathrm{wt}_{\lambda}\left(M_{i}^{\prime}\right) & <\left(1+\frac{g-1}{N+1}\right) m^{2}+\frac{1}{N+1} \sum_{i=1}^{n} m_{i}^{\prime}-\frac{g-1}{N+1} m \\
& =\left(1+\frac{g-1+b}{N+1}\right) m^{2}-\frac{g-1}{N+1} m .
\end{aligned}
$$

This is easily checked; full details are given in Swin, Lemma 2.2.2.

Note that property (i) above requires a set of monomials in $H^{0}\left(\mathbf{P}^{N}, \mathcal{O}_{\mathbf{P}^{N}}(m)\right)$ which map to a basis of $H^{0}\left(C, \mathcal{O}_{C}(m)\right)$ of small weight. We want to turn things around, and instead start on the curve in $H^{0}\left(C, \mathcal{O}_{C}(m)\right)$ and work our way back to $H^{0}\left(\mathbf{P}^{N}, \mathcal{O}_{\mathbf{P}^{N}}(m)\right)$. The action of a 1-PS $\lambda$ of $S L(V)$ on the Hilbert point of a curve induces a weight on elements of $H^{0}\left(C, \mathcal{O}_{C}(m)\right)$ (cf. [HM], p. 208) as follows: take a basis of $H^{0}\left(\mathbf{P}^{N}, \mathcal{O}_{\mathbf{P}^{N}}(1)\right)$ diagonalizing the $\lambda$ action. There is an obvious way to define the weight of any degree $m$ monomial. Then the weight of any degree $m$ homogeneous polynomial is defined as the maximum weight of its constituent monomials, and the weight of an element of $H^{0}\left(C, \mathcal{O}_{C}(m)\right)$ is the minimum of the weights of its preimages in $H^{0}\left(\mathbf{P}^{N}, \mathcal{O}_{\mathbf{P}^{N}}(m)\right)$.

The next proposition says that to establish GIT-stability, it is enough to show that there exists any basis of $H^{0}\left(C, \mathcal{O}_{C}(m)\right)$ of small weight.

Lemma 1.3. If there exist a basis of $H^{0}\left(C, \mathcal{O}_{C}(m)\right)$ of $\lambda$-weight $W$ and monomials $M_{1}^{\prime}, \ldots, M_{n}^{\prime}$ satisfying condition (ii) above, and together these satisfy

$$
W+\sum_{i=1}^{n} \mathrm{wt}_{\lambda} M_{i}^{\prime} \leq\left(1+\frac{g-1+b}{N+1}\right) m^{2}-\frac{g-1}{N+1} m,
$$


then there are monomials $M_{1}, \ldots, M_{P(m)}$ which together with $M_{1}^{\prime}, \ldots, M_{n}^{\prime}$ satisfy conditions (i), (ii), and $\left(i i i^{\prime}\right)$ of the numerical criterion.

This can be proven by a row-reducing argument; see Swin, Lemma 2.2.3 for full details.

1.3. Profiles. Our main tool for computing the weight of a basis is something we call a profile. (Gieseker uses profiles in his proof, but does not name them.) We now define this abstractly.

Let $V$ be a vector space such that every element of $V$ has a weight associated to it. Let $F_{\bullet}$ be a decreasing weighted filtration on $W$. That is, $V=F_{0} \supset F_{1} \supset \cdots \supset$ $F_{N}=0$, and there is a (finite) decreasing sequence of weights $r_{0}>r_{1}>\cdots>r_{N}=$ 0 such that all the elements of $F_{h}$ have weight less than or equal to $r_{h}$.

Definition 1.4. The profile of a decreasing weighted filtration $F_{\bullet}$ as described above is the graph of the decreasing step function in the (codimension $\times$ weight)plane whose value is $r_{h}$ over the interval $\left[\operatorname{codim} F_{h}, \operatorname{codim} F_{h+1}\right)$.

Given a profile, it is possible to choose a basis whose weight is no greater than the area under the profile. We will sometimes speak of the "weight of a filtration" or "weight of a profile". By this we mean the area underneath the profile, which is a bound for the weight of a basis adapted to this filtration. We use this to bound Mumford's $\mu^{L}(x, \lambda)$, since the weight of any basis gives a bound for the minimal weight of a monomial basis.

There is an absolute weight filtration defined as follows: for each possible weight $r_{h}$, form

$$
\Omega\left(r_{h}\right):=\operatorname{Span}\left\{v: v \in V, \operatorname{wt}(v) \leq r_{h}\right\} .
$$

Then the profile associated to $\Omega_{\text {• }}$ can be used to choose a basis of minimum weight, as it tells exactly how many elements of high weight must be added to the basis before elements of lower weight may be added.

\section{A REVIEW OF GIESEKER's PROOF}

Let us quickly review Gieseker's proof from Gies, viewing it as the $n=0$ case of the above setup. We have recast the numerical criterion to say: the $m^{\text {th }}$ Hilbert point of a smooth curve is GIT-stable if and only if there exists a basis of $H^{0}\left(C, \mathcal{O}_{C}(m)\right)$ such that the sum of its weights is less than $(1+\epsilon) m^{2}$.

As discussed before Lemma 1.3, the action of a 1-PS $\lambda$ of $S L(N+1)$ on the Hilbert point of a curve induces weights on elements of $H^{0}\left(C, \mathcal{O}_{C}(m)\right)$ (cf. [HM], p. 208). It is probably most natural to consider the absolute weight filtration on $H^{0}\left(C, \mathcal{O}_{C}(m)\right)$. If one could compute its profile, then one could compute Mumford's function $\mu^{L}(x, \lambda)$ on the nose. However, this is difficult to compute, so Gieseker considers another filtration instead.

Here is a brief and slightly simplified description of the weighted filtration $\widetilde{G}$. Gieseker uses and its profile. Given: a curve $C \subset \mathbf{P}^{N}$ and a 1-PS $\lambda$. As before, renormalize the $\lambda$-weights so that they are decreasing and sum to 1 . Let $\left\{w_{i}\right\}$ be a basis of $H^{0}\left(C, \mathcal{O}_{C}(1)\right) \cong H^{0}\left(\mathbf{P}^{N}, \mathcal{O}(1)\right)$ diagonalizing the $\lambda$ action (and compatible with the order of the $\left.r_{i}\right)$. Let $V_{i}:=\operatorname{Span}\left(\left\{w_{j} \mid j \geq i\right\}\right) \subseteq V$. The normalization of the weights discussed before Lemma 1.3 ensures that all the points $\left(i m, r_{i} m\right)$ 
lie in the first quadrant. Form the lower envelope of these points, and let $0=$ $i_{0}, i_{1}, \ldots$, index the subsequence of points lying on the lower envelope. Then in $H^{0}\left(\mathbf{P}^{N}, \mathcal{O}_{\mathbf{P}^{N}}(m)\right) \cong \operatorname{Sym}^{m} V$ we have the following filtration:

(3)

$$
\begin{array}{cccccccccccc}
\operatorname{Sym}^{m} V=V_{i_{0}}^{m} V_{i_{1}}^{0} & \supset & V_{i_{0}}^{m-1} V_{i_{1}}^{1} & \supset & \cdots & \supset & V_{i_{0}}^{m-p} V_{i_{1}}^{p} & \supset & \cdots & \supset & V_{i_{0}}^{0} V_{i_{1}}^{m}, \\
V_{i_{1}}^{m} V_{i_{2}}^{0} & \supset & V_{i_{1}}^{m-1} V_{i_{2}}^{1} & \supset & \cdots & \supset & V_{i_{1}}^{m-p} V_{i_{2}}^{p} & \supset & \cdots & \supset & V_{i_{1}}^{0} V_{i_{2}}^{m},
\end{array}
$$

etc.

The image of this filtration under restriction to the curve gives a filtration $\widetilde{G}$. of $H^{0}\left(C, \mathcal{O}_{C}(m)\right)$. We can compute the dimension of each stage of the filtration in $H^{0}\left(C, \mathcal{O}_{C}(m)\right)$, and we know the weight of each stage, so this is the data of a profile. The profile is the graph of a step function; its left endpoints lie on the lower envelope of the set of points $\left\{\left(i m, r_{i} m\right)\right\}$. Looking ahead, the lower envelope here is the inspiration for what we will later call the virtual profile.

Any basis adapted to this filtration will establish stability, as the area $A$ under the profile is very close to the area under the lower envelope, and the area under the lower envelope is less than $m^{2}$, by a combinatorial lemma due to Morrison ([Morr], Section 4).

2.1. The weighted filtration on $H^{0}(C, \mathcal{O}(1))$. The action of the 1-PS $\lambda$ induces most fundamentally a weighted filtration on $H^{0}(C, \mathcal{O}(1))$, but to establish stability we need to find a basis of $H^{0}\left(C, \mathcal{O}_{C}(m)\right)$ of small weight. We will be moving between these two vector spaces throughout the rest of the proof. We begin with $H^{0}(C, \mathcal{O}(1))$, and see what our knowledge of this filtration tells us about filtrations on $H^{0}\left(C, \mathcal{O}_{C}(m)\right)$. Once we find formulas for the area under the profile for a certain filtration on $H^{0}\left(C, \mathcal{O}_{C}(m)\right)$, we will ultimately bound the weight of the basis by relating quantities back to their counterparts in $H^{0}(C, \mathcal{O}(1))$.

Let $V_{\bullet}$ be the weighted filtration on $H^{0}(C, \mathcal{O}(1))$ induced by the action of the 1-PS $\lambda$. That is, the stages of the filtration are distinguished by decreasing weight. Let $z_{j}$ be the size of the $j^{\text {th }}$ stage of the filtration, so $z_{j}=\operatorname{codim} V_{j+1}-\operatorname{codim} V_{j}$, and let $r_{j}$ be the weight. Assume that the weights $r_{j}$ have been normalized so that they are decreasing to zero and sum to 1 (that is, $r_{N}=0$ and $\sum z_{j} r_{j}=1$ ). Let $D_{j}$ be the base locus of the sublinear series $V_{j}$, and let $d_{j}=\operatorname{deg} D_{j}$. Let $Q_{1}, \ldots, Q_{q}$ be the points in $\operatorname{Supp} D_{N}$. (It will be convenient to order these points, but the particular choice of ordering does not matter.) The marked points $P_{i}$ may or may not coincide with the $Q$ 's; set

$$
B_{i}=\left\{\begin{array}{l}
\sum_{P_{k}=Q_{i}} b_{k}, \\
0,
\end{array} Q_{i} \neq P_{k} \text { for any } k .\right.
$$

Let $c_{j, i}$ be the multiplicity of $Q_{i}$ in $D_{j}$. (Note that the indices $j, i$ are not in alphabetical order, the opposite of the usual convention.) In general $V_{j}$ is contained in but not equal to $H^{0}\left(C, \mathcal{O}(1)\left(-D_{j}\right)\right)$.

2.2. Relating codegrees and codimensions in $H^{0}(C, \mathcal{O}(1))$. We have one obvious bound on the weights: $\sum z_{j} r_{j}=1$. We will need to relate codegrees $d_{j}=\sum_{i=1}^{q} c_{j, i}$ and codimensions $\sum_{\tau=0}^{j-1} z_{\tau}$.

Near the top of the weighted filtrations the base loci have low degree, so $\mathcal{O}(1)\left(-D_{j}\right)$ has high degree, and the dimension/codimension of $H^{0}\left(C, \mathcal{O}(1)\left(-D_{j}\right)\right)$ may be computed using Riemann-Roch. More precisely: if $\operatorname{deg} D_{j}>d-2 g+1$, then $\operatorname{codim} V_{j}>N-g$. So if $\operatorname{codim} V_{j} \leq N-g$, then $\operatorname{deg} D_{j} \leq d-2 g+1$, so 
$\operatorname{deg} \mathcal{O}(1)\left(-D_{j}\right)>2 g-2$, and $h^{1}\left(\mathcal{O}(1)\left(-D_{j}\right)\right)=0$. Since $V_{j} \subseteq H^{0}\left(C, \mathcal{O}(1)\left(-D_{j}\right)\right)$, we get a bound: the codegree of $\mathcal{O}(1)\left(-D_{j}\right)$ cannot exceed the codimension of $V_{j}$. From the definition of $z_{j}$ and $c_{j, i}$ above we have codim $V_{j}=\sum_{\tau=0}^{j-1} z_{\tau}$ and $\operatorname{deg} D_{j}=\sum_{i=1}^{q} c_{j, i}$. We thus obtain:

$$
\text { if } \sum_{\tau=0}^{j-1} z_{\tau} \leq N-g \text {, then } \sum_{i=1}^{q} c_{j, i} \leq \sum_{\tau=0}^{j-1} z_{\tau} .
$$

We call this the Riemann-Roch region of the filtration. Write $j_{R R}$ for the largest index $j$ which satisfies $\sum_{\tau=0}^{j-1} z_{\tau} \leq N-g$.

On the other hand, if $\mathcal{O}(1)$ itself is special, or for stages of the filtration of high codimension (that is, near the bottom) the line bundles $\mathcal{O}\left(-D_{j}\right)$ have low degree, then we might have $h^{1}\left(\mathcal{O}\left(-D_{j}\right)\right) \neq 0$. Here we can use Clifford's Theorem to get the following bound:

$$
\text { if } \begin{aligned}
& \sum_{\tau=0}^{j-1} z_{\tau}>N-g, \text { then } \sum_{i=1}^{q} c_{j, i} \leq \sum_{\tau=0}^{j-1} z_{\tau} \\
& +\left(\sum_{\tau=0}^{j-1} z_{\tau}-(N-g)\right)-h^{1}(C, \mathcal{O}(1)) .
\end{aligned}
$$

We call this the Clifford region of the filtration and write $j_{\text {Cliff }}$ for the smallest index $j$ which satisfies $\sum_{\tau=0}^{j-1} z_{\tau}>N-g$. (Thus $j_{C l i f f}=j_{R R}+1$.)

2.3. Passing to $H^{0}\left(C, \mathcal{O}_{C}(m)\right)$. We want to use the base loci $D_{j}$ to control how multiples of the $V_{j}$ intersect, and this would work best if $V_{j}=H^{0}\left(C, \mathcal{O}(1)\left(-D_{j}\right)\right)$. Gieseker observed that if we pass from $H^{0}(C, \mathcal{O}(1))$ to $H^{0}\left(C, \mathcal{O}_{C}(m)\right.$ ) (which is where we ultimately need to produce a basis anyway), then we will be able to treat an arbitrary 1-PS $\lambda$ as if it were of this form.

Let $\left(V_{s}^{u-w} V_{t}^{w} V_{0}\right)^{v}$ denote the subspace of $H^{0}(C, \mathcal{O}((u+1) v))$ generated by expressions of the form $x_{1} \cdots x_{v(u-w)} y_{1} \cdots y_{v w} z_{1} \cdots z_{v}$, where the $x$ 's come from $V_{s}$, the $y$ 's come from $V_{t}$, and the $z$ 's come from $V_{0}$.

Lemma 2.1 (Gieseker's Multiplication Lemma). Let $u, v, w$ be nonnegative integers with $0 \leq w \leq u$ and $v \geq 1$. Suppose $C$ is an arbitrary subscheme of $\mathbf{P}^{N}$ with Hilbert polynomial $d t-g+1$ and

$$
v \geq \frac{d^{2}(u+1)^{2}-d(u+1)}{2}-g+1 .
$$

Then

$$
\left(V_{s}^{u-w} V_{t}^{w} V_{0}\right)^{v}=H^{0}\left(C, \mathcal{O}((u+1) v)\left(-(u-w) D_{s}-w D_{t}\right)\right)
$$

Remark. Note that the bound on $v$ depends on $u$ and the Hilbert polynomial $P(z)=d z-g+1$, but not on the curve $C$ or the line bundle $\mathcal{O}_{C}(1)$ embedding $C$ into $\mathbf{P}^{N}$.

Proof. Most of the proof of Lemma 2.1 comes from pages 54-55 of G2] we need only add a few comments. First, let $L_{s}$ be the line bundles generated by the sections in $V_{s}$; then $L_{s}=\mathcal{O}_{C}(1)\left(-D_{s}\right)$. Carrying on in this fashion gives us the right hand side of (7). Second, the explicit bound on $v$ comes from computing Gotzmann numbers for the embeddings in Gieseker's proof. See Swin, Lemma 2.3.1 for full details.

Let $m=(u+1) v$. Then $H^{0}\left(C, \mathcal{O}_{C}(m)\right)$ is filtered by the subspaces $\left(V_{j}^{u} V_{0}\right)^{v}$. 
Note that if there are two successive stages in $V_{\bullet}$ where the base locus does not increase, the images of these two stages are the same upon passing to $H^{0}\left(C, \mathcal{O}_{C}(m)\right)$. Thus, we will want to record the subsequence of the $j$ 's where the degree of the base locus increases. We will subindex these by the letter $k$ and we will simply write $k$ rather than writing $j_{k}$.

We define a filtration $\widetilde{V} \bullet$ of $H^{0}\left(C, \mathcal{O}_{C}(m)\right)$ as follows:

$$
\widetilde{V}_{(k, w)}=\left(V_{k}^{u-w} V_{k+1}^{w} V_{0}\right)^{v} .
$$

The filtration $\widetilde{V}_{\bullet}$ is indexed by pairs $(k, w)$ in lexicographic order. We will use tildes for quantities associated to $\widetilde{V}_{\bullet}$. We have $\widetilde{V}_{(k, 0)}=H^{0}\left(C, \mathcal{O}(m)\left(-\widetilde{D}_{k}\right)\right)$, where $\widetilde{D}_{k}=u v D_{j_{k}}$. We write $\widetilde{d}_{k}:=u v d_{j_{k}}$ and $\widetilde{c}_{k, i}:=u v c_{j_{k}, i}$. Then

$$
\widetilde{V}_{(k, 0)}=H^{0}\left(C, \mathcal{O}(m)\left(-\widetilde{c}_{k, 1} Q_{1}-\cdots-\widetilde{c}_{k, q} Q_{q}\right)\right),
$$

and elements of this space have weight $\leq \widetilde{r}_{k}:=u v r_{j_{k}}+v r_{0}$.

Define $\widetilde{N}$ to be the smallest index giving the $v r_{0}$-weight space. We have:

$$
\begin{array}{lc}
\frac{\text { Space }}{\widetilde{V}_{(0,0)}}=H^{0}\left(C, \mathcal{O}_{C}(m)\right) & \frac{\text { Weight }}{\widetilde{r}_{0}} \\
\widetilde{V}_{(1,0)}=H^{0}\left(C, \mathcal{O}(m)\left(-\widetilde{c}_{1,1} Q_{1}-\cdots-\widetilde{c}_{1, q} Q_{q}\right)\right) & \widetilde{r}_{1} \\
\widetilde{V}_{(2,0)}=H^{0}\left(C, \mathcal{O}(m)\left(-\widetilde{c}_{2,1} Q_{1}-\cdots-\widetilde{c}_{2, q} Q_{q}\right)\right) & \widetilde{r}_{2} \\
\vdots & \vdots \\
\widetilde{V}_{(\widetilde{N}, 0)}=H^{0}\left(C, \mathcal{O}(m)\left(-\widetilde{c}_{\widetilde{N}, 1} Q_{1}-\cdots-\widetilde{c}_{\widetilde{N}, q} Q_{q}\right)\right) & \widetilde{r}_{\widetilde{N}}=v r_{0}
\end{array}
$$

We may extract the multiplicities of the points in the base loci in the weighted filtration $\widetilde{V}_{\bullet}$ and the weights to obtain an $(\widetilde{N}+1) \times(q+1)$ array:

$$
\left(\begin{array}{cccc}
\widetilde{c}_{0,1} & \cdots & \widetilde{c}_{0, q} & \widetilde{r}_{0} \\
\widetilde{c}_{1,1} & \cdots & \widetilde{c}_{1, q} & \widetilde{r}_{1} \\
\vdots & \vdots & \vdots & \vdots \\
\widetilde{c}_{\widetilde{N}, 1} & \cdots & \widetilde{c}_{\widetilde{N}, q} & \widetilde{r}_{\widetilde{N}}=v r_{0}
\end{array}\right) .
$$

This array has the following properties: the $\widetilde{c}_{k, i}$ 's are all nonnegative integers, the $\widetilde{r}_{i}$ 's are rational numbers weakly decreasing to $v r_{0}$, and in the first row the $\widetilde{c}_{0, i}$ 's are all zero. Furthermore, we see that the sum of the first $q$ entries in row $k$ is governed by either a Riemann-Roch bound (5) or a Clifford bound (6).

\section{Why GIESEKER's PROOF DOESN'T COVER MARKED POINTS}

To our knowledge, Elizabeth Baldwin first wrote the straightforward generalization of Gieseker's result to $\bar{M}_{g, n}$ (unpublished), and it is not difficult to see that the analogue of Gieseker's filtration does not suffice to establish stability in cases where $b_{i}$ is more than a little larger than 0 . Here is a counterexample:

3.1. Example 1. This example shows that the profile associated to $\widetilde{G}_{\bullet}$ (which equals $\widetilde{V}_{\bullet}$ in this example) does not suffice to establish asymptotic Hilbert stability when there are marked points. 
Suppose $n \geq 3$. Consider the 1-PS $\lambda$ which acts with linearly decreasing weights on the marked points. That is, $\lambda$ induces the following weighted filtration:

$\begin{array}{rlr}\frac{\text { Space }}{V_{0}} & =H^{0}(C, \mathcal{O}(1)) & \frac{\text { Weight }}{\frac{1}{2}} \\ V_{1} & =H^{0}\left(C, \mathcal{O}(1)\left(-P_{1}\right)\right) & \frac{1}{3} \\ V_{2} & =H^{0}\left(C, \mathcal{O}(1)\left(-P_{1}-P_{2}\right)\right) & \frac{1}{6} \\ V_{3} & =H^{0}\left(C, \mathcal{O}(1)\left(-P_{1}-P_{2}-P_{3}\right)\right) & 0\end{array}$

The points $\left(i m, r_{i} m\right)$ all lie on their lower envelope. Also, we have $r_{0}+r_{1}+r_{2}=1$. Using $b_{1}=b_{2}=b_{3}=1 / 2$, we have $T \approx A m^{2}+\sum_{i=1}^{3} b_{i} r_{i} m^{2}=3 / 4 m^{2}+1 / 2 m^{2}=$ $5 / 4 m^{2}>(1+\epsilon) m^{2}$.

In this example we chose a specific linearization $b_{1}=b_{2}=b_{3}=1 / 2$. However, this is not an isolated example. Indeed, we see from the calculation above that Gieseker's estimates will not establish stability for any set of linearizing weights such that $\sum_{i=1}^{3} b_{i} r_{i}>1 / 4+\epsilon$, or equivalently, $3 b_{1}+2 b_{2}+b_{3}>3 / 2+\epsilon$. More generally, if there are $n$ marked points, then by using a 1-PS analogous to that used above (that is, a 1-PS for which the base locus increases by $P_{i}$ at each stage of the filtration, while the weights decrease linearly) one can show that Gieseker's estimates will not suffice whenever $\sum_{i=1}^{n} b_{i}(2-i / n)>1$. In particular, if the weights $b_{i}$ are symmetric, say each $b_{i}=\bar{b}$, then Gieseker's estimates fail if $\bar{b}>2 /(3 n-1)$.

In Swin, Section 3.4, the linearizations used to construct the moduli spaces $\bar{M}_{g, \mathcal{A}}$ are of the form $b_{i} \approx a_{i} / 2$. Thus, the straightforward adaptation of Gieseker's proof is not enough to establish the stability of smooth pointed curves for a large range of important linearizations.

3.2. Minimizing multiplicities. First, we recall an easy linear algebra lemma:

Lemma 3.1. Let $V_{1}, \ldots, V_{n}$ be subspaces of a vector space $V$. Write $V_{i j}:=V_{i} \cap V_{j}$, $V_{i j k}:=V_{i} \cap V_{j} \cap V_{k}$, etc. Then

$$
\begin{aligned}
& \operatorname{codim} \operatorname{Span}\left\{V_{1}, \ldots, V_{n}\right\} \\
& =\sum \operatorname{codim} V_{i}-\sum_{i<j} \operatorname{codim} V_{i j}+\sum_{i<j<k} \operatorname{codim} V_{i j k}-\cdots+(-1)^{n-1} \operatorname{codim} V_{123 \cdots n} .
\end{aligned}
$$

Soon we are going to put a lot of effort into minimizing multiplicities. The following lemma shows that this makes easy work of computing spans of spaces of the form we have encountered.

Lemma 3.2 (Span Lemma). Suppose we are given $q$ subspaces $E_{1}, \ldots, E_{q}$ of $H^{0}\left(C, \mathcal{O}_{C}(m)\right)$ of the form:

$$
\begin{aligned}
E_{1} & =H^{0}\left(C, \mathcal{O}(m)\left(-d_{1,1} Q_{1}-\cdots-d_{1, q} Q_{q}\right)\right), \\
E_{2} & =H^{0}\left(C, \mathcal{O}(m)\left(-d_{2,1} Q_{1}-\cdots-d_{2, q} Q_{q}\right)\right) \\
& \vdots \\
E_{q} & =H^{0}\left(C, \mathcal{O}(m)\left(-d_{q, 1} Q_{1}-\cdots-d_{q, q} Q_{q}\right)\right) .
\end{aligned}
$$

The $E_{i}$ need not be distinct, and although the notation looks a little similar to that of filtrations above, we do not mean in any way to imply that the $E_{i}$ form a filtration -in the applications we have in mind, they do not. 
Suppose that $E_{i}$ minimizes the multiplicity of $Q_{i}$ - that is, the minimum in each column appears along the diagonal. Suppose also that

$$
\sum_{i=1}^{q} \max _{j} d_{j, i}<d m-2 g
$$

Then

$$
\operatorname{Span}\left(E_{1}, \ldots, E_{q}\right)=H^{0}\left(C, \mathcal{O}(m)\left(-\sum_{i=1}^{q} d_{i, i} Q_{i}\right)\right)
$$

and

$$
\operatorname{codim} \operatorname{Span}\left(E_{1}, \ldots, E_{q}\right)=d_{1,1}+d_{2,2}+\cdots+d_{q, q} .
$$

Proof. The condition

$$
\sum_{i=1}^{q} \max _{j} d_{j, i} Q_{i}<d m-2 g
$$

ensures that the codimension of the intersection of any subset of these $q$ spaces may be computed using Riemann-Roch. Thus, for each subset $I \subseteq\{1, \ldots, q\}$, say $I=\left\{i_{1}, \ldots, i_{k}\right\}$, we have

$\operatorname{codim} E_{i_{1} \cdots i_{k}}=\max \left(d_{i_{1}, 1}, \ldots, d_{i_{k}, 1}\right)+\max \left(d_{i_{1}, 2}, \ldots, d_{i_{k}, 2}\right)+\cdots+\max \left(d_{i_{1}, q}, \ldots, d_{i_{k}, q}\right)$.

Suppose $j \notin I$. Then we claim that in (11) the term $\max \left(d_{i_{1}, j}, \ldots, d_{i_{k}, j}\right)$ is cancelled by a term coming from $I \cup\{j\}$. Being a subset of cardinality greater than one, the codimension of the intersection indexed by $I \cup\{j\}$ gets an opposite sign from that indexed by $I$. Also, since by hypothesis $d_{j, j}$ is the smallest term in column $j$, it drops out of $\max \left(d_{i_{1}, j}, \ldots, d_{i_{k}, j}, d_{j, j}\right)$, giving us exactly the cancellation we claimed. Given $I$, every $j \in\{1, \ldots, q\}$ is either in $I$ or not in $I$, so it is clear whether the term $\max \left(d_{i_{1}, j}, \ldots, d_{i_{k}, j}\right)$ is cancelling or being cancelled. The only terms surviving are the $d_{i, i}$ since there are no double intersections of the form $E_{i i}$ in our setup to cancel them.

Finally, the base locus of $\operatorname{Span}\left(E_{1}, \ldots, E_{q}\right)$ must be $\sum_{i=1}^{q} d_{i, i} Q_{i}$ (since we can find sections that vanish to each $Q_{i}$ to exactly order $\left.d_{i, i}\right)$. This gives

$$
\operatorname{Span}\left(E_{1}, \ldots, E_{q}\right) \subset H^{0}\left(C, \mathcal{O}(m)\left(-\sum_{i=1}^{q} d_{i, i} Q_{i}\right)\right) .
$$

But these two spaces have the same codimension, so they are equal.

\section{The Filtration $\tilde{X} \bullet$ And its profile}

4.1. Subscript conventions. In the course of the proof we will need to keep track of a set of subsequences of a subsequence of a sequence. We use the following notation and conventions.

4.1.1. Tildes. Tildes are used when we are working in $H^{0}\left(C, \mathcal{O}_{C}(m)\right)$. Recall from Section 2 that quantities associated to $V_{\bullet}$ (such as the multiplicities $c_{\bullet}, i$ and the weights $r_{\bullet}$ ) have no tildes and are fundamentally indexed by $j$ 's. Quantities associated to $\widetilde{V}_{\bullet}$ (such as $\widetilde{c}_{\bullet}, i$ and weights $\widetilde{r}_{\bullet}$ ) are written with tildes and indexed by $k$ 's, where $k$ indexes the subsequence of the rows $j$ of the original filtration $V$ • where the base locus increases. 
4.1.2. Avoiding nested subscripts. When we want to refer to the subsequence of $c_{\bullet, i}$ or $r_{\bullet}$ corresponding to stages of $V_{\bullet}$ where the base locus increases, rather than using nested subscripts and writing for instance $r_{j_{k}}$, we will simply write $r_{k}$.

4.1.3. Cases $I-I V$ and the functions $s(k, i)$ and $t(k, i)$. It is useful to define two functions $s$ and $t$. We will take the time now to define four cases, which will be referred to in this section and in Section 5

I. We have $\widetilde{c}_{k, i}<\widetilde{c}_{k+1, i}<\widetilde{c}_{k+2, i}$. That is, the multiplicity of the point $Q_{i}$ jumps at row $k$ and again at row $k+1$. In this case we define $s(k, i)=k$ and $t(k, i)=k+1$.

II. We have $\widetilde{c}_{k, i}=\widetilde{c}_{k+1, i}=\widetilde{c}_{k+2, i}$. That is, the multiplicity of $Q_{i}$ does not jump at row $k$ or at row $k+1$. Define $s(k, i)$ to be the last row where this multiplicity jumped, and let $t(k, i)$ be the next row where it jumps, or else $\widetilde{N}$ if $\widetilde{c}_{k, i}=\widetilde{c}_{\widetilde{N}, i}$. In symbols, in Case II, $s(k, i)$ is the largest index strictly less than $k$ such that $\widetilde{c}_{s(k, i), i}<\widetilde{c}_{s(k, i)+1, i}$, and $t(k, i)$ is the smallest index strictly greater than $k$ such that $\widetilde{c}_{t(k, i), i}<\widetilde{c}_{t(k, i)+1, i}$ if this exists, or else $\widetilde{N}$.

III. We have $\widetilde{c}_{k, i}=\widetilde{c}_{k+1, i}<\widetilde{c}_{k+2, i}$. That is, the multiplicity of $Q_{i}$ does not jump at row $k$ but jumps at row $k+1$. Then as in Case II we define $s(k, i)$ to be the last row where this multiplicity jumped, and we define $t(k, i)=k+1$.

IV. We have $\widetilde{c}_{k, i}<\widetilde{c}_{k+1, i}=\widetilde{c}_{k+2, i}$. That is, the multiplicity of $Q_{i}$ jumps at row $k$ but not at row $k+1$. We define $s(k, i)=k$, and as in Case II let $t(k, i)$ be the next row where this multiplicity jumps, or else $\widetilde{N}$ if $\widetilde{c}_{k, i}=\widetilde{c}_{\widetilde{N}, i}$.

It follows from the definitions of Cases II, III, and IV that if row $k$ is in Case II, then we have $s(k+1, i)=s(k, i)$ and $t(k+1, i)=t(k, i)$.

Defining $s$ and $t$ differently in Cases I-IV as we have done permits us to treat these cases simultaneously in Section 5.2 , which more than makes up for the extra work involved here.

4.1.4. Eliminating redundancies. Rather than printing $i$ redundantly in subscripts, whenever we can we will omit it the second time. For example we will simply write $\widetilde{c}_{s(k, i)}$ for $\widetilde{c}_{s(k, i), i}$.

4.1.5. The functions $j(i, \ell)$ and $k(i, \ell)$. We will also want to keep track of the subset of $j$ 's or $k$ 's where the multiplicity of the point $Q_{i}$ in the base locus increases. We will do this as follows:

Say the multiplicity of $Q_{i}$ jumps $K_{i}$ times between the top of the filtration and the bottom. We start counting from zero, so these stages of the filtration are the $0^{\text {th }}$ jump up through the $\left(K_{i}-1\right)^{t h}$ jump. As a convention, we append $\bar{N}$ (the index of the last row of the filtration $V_{\bullet}$ ) or $\widetilde{N}$ (the index of the last row of the filtration $\left.\widetilde{V}_{\bullet}\right)$ as the $K_{i}^{t h}$ element of this sequence. We write two increasing set functions

$$
j(i, \bullet):\left\{0, \ldots, K_{i}\right\} \rightarrow\{0, \ldots, \bar{N}\}
$$

and

$$
k(i, \bullet):\left\{0, \ldots, K_{i}\right\} \rightarrow\{0, \ldots, \widetilde{N}\}
$$

and use these to index the rows where the multiplicity of the point $Q_{i}$ in the base locus increases. That is, the function $j(i, \bullet)$ takes values in the $j$ 's, and similarly $k(i, \bullet)$ takes values in the $k$ 's. Here is an example to give a little practice with this 
notation: $j(i, 0)$ means the index $j$ where the multiplicity of $Q_{i}$ jumps for the $0^{t h}$ time. This is the lowest row of the filtration where $Q_{i}$ is not in the base locus, so $r_{j(i, 0)}$ is the least weight of a section not vanishing at $Q_{i}$.

As before, when $i$ appears more than once in a subscript, we will omit it the second time. Thus $c_{j(i, 0), i}$ becomes $c_{j(i, 0)}$, and we have $c_{j(i, 0)}=0$ while $c_{j(i, 0)+1}=$ $c_{j(i, 1)}>0$.

4.1.6. A consequence of these conventions. As a consequence, note that previously when going between the filtrations $V_{\bullet}$ and $\widetilde{V}_{\bullet}$ we had $\widetilde{c}_{k, i}=u v c_{j_{k}, i}$. But now with our new notation we can write $\widetilde{c}_{k(i, \ell)}=u v c_{j(i, \ell)}$. In this sense the definitions of $j(i, \ell)$ and $k(i, \ell)$ have eliminated some of the need for nested subscripts.

4.2. The filtration $\tilde{X}_{\bullet}$ and its profile. Here we describe the filtration $\tilde{X}_{\bullet}$ of $H^{0}\left(C, \mathcal{O}_{C}(m)\right)$ and its weight profile. $\widetilde{X}_{\bullet}$ is obtained from the filtration $\widetilde{V}_{\bullet}$ by taking spans of the stages of $\widetilde{V}_{\bullet}$ with other cleverly chosen spaces.

Like $\widetilde{V}_{\bullet}$, the filtration $\widetilde{X}_{\bullet}$ has $(\widetilde{N} \times u)+1$ stages and is indexed by pairs $(k, w)$, ordered lexicographically.

For each $k=0, \ldots, \widetilde{N}-1$ and for each $w=0, \ldots, u-1$ we want to describe the space $\widetilde{X}_{k, w}$. Our starting point is the space $\left(V_{k}^{u-w} V_{k+1}^{w} V_{0}\right)^{v}$. Elements of this space have weight less than or equal to $v(u-w) r_{k}+v w r_{k+1}+v r_{0}$.

Our goal: for each $i$ from 1 to $q$, find a subspace of $H^{0}\left(C, \mathcal{O}_{C}(m)\right)$ such that

(1) the weight is less than or equal to the weight of $\left(V_{k}^{u-w} V_{k+1}^{w} V_{0}\right)^{v}$,

(2) the multiplicity of $Q_{i}$ in its base locus is less than the multiplicity of $Q_{i}$ in the base locus of $\left(V_{k}^{u-w} V_{k+1}^{w} V_{0}\right)^{v}$.

We do this as described in the following definition. Also, it is convenient to define certain quantities $\widetilde{x}(k, w, i)$ at this time; their role will be explained soon.

Definition 4.1 (The filtration $\tilde{X}_{\bullet}$ and its profile).

First, $\widetilde{X}_{0,0}=H^{0}\left(C, \mathcal{O}_{C}(m)\right)$.

For the remaining triples $(k, w, i)$ with $(k, w) \neq(0,0)$, where $k=0, \ldots, \tilde{N}-1$, $w=0, \ldots, u-1$, we begin with $\left(V_{k}^{u-w} V_{k+1}^{w} V_{0}\right)^{v}$. For each $i=1, \ldots, q$, there may be an additional contribution to the profile as follows:

- If the multiplicity of $Q_{i}$ is zero in row $k+1$ (and hence zero in row $k$ also), there is no contribution to $\widetilde{X}_{k, w}$, and $\widetilde{x}(k, w, i)=0$.

- If the multiplicity of $Q_{i}$ is nonzero in row $k+1$ and we are in Case I as defined in Section 4.1.3, so the multiplicity of $Q_{i}$ jumps at row $k$ and row $k+1$, there is no further contribution to $\widetilde{X}_{k, w}$ beyond $\left(V_{k}^{u-w} V_{k+1}^{w} V_{0}\right)^{v}$, and $\widetilde{x}(k, w, i)$ is the multiplicity of $Q_{i}$ in $\left(V_{k}^{u-w} V_{k+1}^{w} V_{0}\right)^{v}$.

- If the multiplicity of $Q_{i}$ is nonzero in row $k+1$ and we are in Case II, III, or IV as defined in Section 4.1.3, so the multiplicity of $Q_{i}$ jumps at no more than one of the rows $k$ and $k+1$, let $s(k, i)$ and $t(k, i)$ be as defined there. For each $w$ we find the smallest integer $W=W(u, v ; k, w, i)$ such that $\left(V_{s(k, i)}^{u-W} V_{t(k, i)}^{W} V_{0}\right)^{v}$ has weight less than $v(u-w) r_{k}+v w r_{k+1}+v r_{0}$. Then $\left(V_{s(k, i)}^{u-W} V_{t(k, i)}^{W} V_{0}\right)^{v}$ is added to $\widetilde{X}_{k, w}$, and $\widetilde{x}(k, w, i)$ is the multiplicity of $Q_{i}$ in the base locus of $\left(V_{s(k, i)}^{u-W} V_{t(k, i)}^{W} V_{0}\right)^{v}$. 
Then define

$\widetilde{X}_{k, w}:=\operatorname{Span}\left\{\left(V_{k}^{u-w} V_{k+1}^{w} V_{0}\right)^{v}\right.$, spaces of type $\left(V_{s(k, i)}^{u-W} V_{t(k, i)}^{W} V_{0}\right)^{v}$ if there are any $\}$, and let $\widetilde{x}(k, w)$ be the codimension of $\widetilde{X}_{k, w}$.

Note $\widetilde{X}_{k, w}$ is the span of between 1 and $q+1$ distinct spaces. There may be fewer than $q+1$ distinct spaces in the span, as there may be points $Q_{i}$ which make no contribution, and/or repeats may occur among the spaces of the form $\left(V_{s(k, i)}^{u-W} V_{t(k, i)}^{W} V_{0}\right)^{v}$.

Finally, for the last stage of the filtration, define $\widetilde{X}_{\widetilde{N}}:=\widetilde{V}_{\widetilde{N}}$.

The profile associated to $\widetilde{X}_{\bullet}$ is the graph of decreasing step function whose value over the intervals $[\widetilde{x}(k, w), \widetilde{x}(k, w+1))$ is $v(u-w) r_{k}+v w r_{k+1}+v r_{0}$, and whose value over the interval $\left[\operatorname{codim} \widetilde{X}_{\tilde{N}}, \operatorname{dim} H^{0}\left(C, \mathcal{O}_{C}(m)\right)\right]$ is $v r_{0}$.

4.2.1. Illustration: The filtration $\tilde{X}_{\bullet}$ and its profile for Example 1. Recall that Example 1 concerns the 1-PS with $n=q=3$, which induces the following weight filtration $V_{\bullet}$ :

$\begin{array}{llr}\frac{\text { Space }}{V_{0}} & =H^{0}(C, \mathcal{O}(1)) & \frac{\text { Weight }}{\frac{1}{2}} \\ V_{1} & =H^{0}\left(C, \mathcal{O}(1)\left(-P_{1}\right)\right) & \frac{1}{3} \\ V_{2} & =H^{0}\left(C, \mathcal{O}(1)\left(-P_{1}-P_{2}\right)\right) & \frac{1}{6} \\ V_{3} & =H^{0}\left(C, \mathcal{O}(1)\left(-P_{1}-P_{2}-P_{3}\right)\right) & 0\end{array}$

After passing to $H^{0}\left(C, \mathcal{O}_{C}(m)\right)$ we obtain the filtration $\widetilde{V}_{\bullet}$ :

$\begin{array}{llcl}\frac{\text { Space }}{\widetilde{V}_{0}} & =H^{0}\left(C, \mathcal{O}_{C}(m)\right) & & \text { Weight } \\ \widetilde{V}_{1} & =H^{0}\left(C, \mathcal{O}(m)\left(-u v P_{1}\right)\right) & \frac{1}{2} u v+\frac{1}{2} v \\ \widetilde{V}_{2} & =H^{0}\left(C, \mathcal{O}(m)\left(-u v P_{1}-u v P_{2}\right)\right) & \frac{1}{3} u v+\frac{1}{2} v \\ \widetilde{V}_{3} & =H^{0}\left(C, \mathcal{O}(m)\left(-u v P_{1}-u v P_{2}-u v P_{3}\right)\right) & \frac{1}{2} v\end{array}$

We compute the filtration $\tilde{X}_{\bullet}$ and its profile for Example 1. For this, we ought to specify $u, v$ first. We choose $u=3$ and $v=5$. (This value of $v$ is too small to satisfy the hypotheses of Lemma 2.1. but let us ignore this in the interest of presenting a reasonably sized example.) Also, in this example, we will always have $\widetilde{x}(k, w)=\sum_{i=1}^{q} \widetilde{x}(k, w, i)$. (See [Swin, pp. 63-64 for an explanation of this equality.)

The filtration $\tilde{X}_{\bullet}$ has ten stages. The first and the last are easy to compute-we have $\widetilde{X}_{0,0}=H^{0}\left(C, \mathcal{O}_{C}(m)\right)$ and $\widetilde{X}_{3}=\left(V_{3}^{3} V_{0}\right)^{5}$. Let's compute one of the middle stages, $\widetilde{X}_{1,1}$, as an example: the multiplicity of $P_{1}$ does not increase from row 1 to row 2 to row 3 , so we are in Case II, and $s(1,1)=0$ and $t(1,1)=3$. We find $W=2$. (Here $W$ may be computed from its defining properties or by skipping ahead and using formula (20) derived in Section 5) Thus the contribution to $\widetilde{X}_{1,1}$ from $P_{1}$ is $\left(V_{0}^{1} V_{3}^{2} V_{0}\right)^{5}$, and $\widetilde{x}(1,1,1)=10$. The multiplicity of $P_{2}$ increases from row 1 to row 2 , but not from row 2 to row 3 , so we are in Case IV, and $s(1,2)=1$ and $t(1,2)=3$. Here $W=1$, and the contribution from $P_{2}$ to $\widetilde{X}_{1,1}$ is $\left(V_{1}^{2} V_{3}^{1} V_{0}\right)^{5}$, and 
$\widetilde{x}(1,1,2)=5$. The multiplicity of $P_{3}$ is zero in both row 1 and row 2 , so $P_{3}$ does not contribute to $\widetilde{X}_{1,1}$. We have: $\widetilde{X}_{1,1}=\operatorname{Span}\left\{\left(V_{1}^{2} V_{2}^{1} V_{0}\right)^{5},\left(V_{0}^{1} V_{3}^{2} V_{0}\right)^{5},\left(V_{1}^{2} V_{3}^{1} V_{0}\right)^{5}\right\}$, and $\widetilde{x}(1,1)=15$.

Here are the filtrations $\widetilde{G}_{\bullet}$ and $\widetilde{X}_{\bullet}$. We have left the spans unsimplified.

\begin{tabular}{|c|c|c|c|c|c|}
\hline$(k, w)$ & wt & $\widetilde{G}_{(k, w)}$ & $\operatorname{codim} \widetilde{G} \bullet$ & $\widetilde{X}_{(k, w)}$ & $\operatorname{codim} \tilde{X} \bullet$ \\
\hline$(0,0)$ & 10 & $\left(V_{0}^{3} V_{1}^{0} V_{0}\right)^{5}$ & 0 & $H^{\mathrm{O}}\left(C, \mathcal{O}_{C}(m)\right)$ & 0 \\
\hline$(0,1)$ & $55 / 6$ & $\left(V_{0}^{2} V_{1}^{1} V_{0}\right)^{5}$ & 5 & $\operatorname{Span}\left\{\left(V_{0}^{2} V_{1}^{1} V_{0}\right)^{5},\left(V_{0}^{2} V_{3}^{1} V_{0}\right)^{5}\right\}$ & 5 \\
\hline$(0,2)$ & $50 / 6$ & $\left(V_{0}^{1} V_{1}^{2} V_{0}\right)^{5}$ & 10 & $\operatorname{Span}\left\{\left(V_{0}^{1} V_{1}^{2} V_{0}\right)^{5},\left(V_{0}^{2} V_{3}^{1} V_{0}\right)^{5}\right\}$ & 5 \\
\hline$(1,0)$ & $45 / 5$ & $\left(V_{1}^{3} V_{2}^{0} V_{0}\right)^{5}$ & 15 & $\begin{array}{c}\operatorname{Span}\left\{\left(V_{1}^{3} V_{2}^{0} V_{0}\right)^{5},\left(V_{0}^{2} V_{3}^{1} V_{0}\right)^{5}\right. \\
\left.\left(V_{1}^{3} V_{3}^{0} V_{0}\right)^{5}\right\}\end{array}$ & 5 \\
\hline$(1,1)$ & $40 / 6$ & $\left(V_{1}^{2} V_{2}^{1} V_{0}\right)^{5}$ & 20 & $\begin{array}{c}\operatorname{Span}\left\{\left(V_{1}^{2} V_{2}^{1} V_{0}\right)^{5},\left(V_{0}^{1} V_{3}^{2} V_{0}\right)^{5},\right. \\
\left.\left(V_{1}^{2} V_{3}^{1} V_{0}\right)^{5}\right\}\end{array}$ & 15 \\
\hline$(1,2)$ & $35 / 6$ & $\left(V_{1}^{1} V_{2}^{2} V_{0}\right)^{5}$ & 25 & $\begin{array}{c}\operatorname{Span}\left\{\left(V_{1}^{1} V_{2}^{2} V_{0}\right)^{5},\left(V_{0}^{1} V_{3}^{2} V_{0}\right)^{5}\right. \\
\left.\left(V_{1}^{2} V_{3}^{1} V_{0}\right)^{5}\right\}\end{array}$ & 15 \\
\hline$(2,0)$ & 5 & $\left(V_{2}^{3} V_{3}^{0} V_{0}\right)^{5}$ & 30 & $\begin{array}{r}\operatorname{Span}\left\{\left(V_{2}^{3} V_{3}^{0} V_{0}\right)^{5},\left(V_{0}^{1} V_{3}^{2} V_{0}\right)^{5}\right. \\
\left.\left(V_{1}^{1} V_{3}^{2} V_{0}\right)^{5},\left(V_{2}^{3} V_{3}^{0} V_{0}\right)^{5}\right\}\end{array}$ & 20 \\
\hline$(2,1)$ & $25 / 6$ & $\left(V_{2}^{2} V_{3}^{1} V_{0}\right)^{5}$ & 35 & $\begin{array}{r}\operatorname{Span}\left\{\left(V_{2}^{2} V_{3}^{1} V_{0}\right)^{5},\left(V_{0}^{0} V_{3}^{3} V_{0}\right)^{5}\right. \\
\left.\left(V_{1}^{1} V_{3}^{2} V_{0}\right)^{5},\left(V_{2}^{2} V_{3}^{1} V_{0}\right)^{5}\right\}\end{array}$ & 30 \\
\hline$(2,2)$ & $20 / 6$ & $\left(V_{2}^{1} V_{3}^{2} V_{0}\right)^{5}$ & 40 & $\begin{array}{r}\operatorname{Span}\left\{\left(V_{2}^{1} V_{3}^{2} V_{0}\right)^{5},\left(V_{0}^{0} V_{3}^{3} V_{0}\right)^{5}\right. \\
\left.\left(V_{1}^{0} V_{3}^{3} V_{0}\right)^{5},\left(V_{2}^{1} V_{3}^{2} V_{0}\right)^{5}\right\}\end{array}$ & 40 \\
\hline 3 & $15 / 6$ & $\left(V_{3}^{3} V_{0}\right)^{5}$ & 45 & $\left(V_{3}^{3} V_{0}\right)^{5}$ & 45 \\
\hline
\end{tabular}

Notice that $\widetilde{X}_{0,1}=\widetilde{X}_{0,2}=\widetilde{X}_{1,0}$, and $\widetilde{X}_{1,1}=\widetilde{X}_{1,2}$. Thus, when we compute the area under the profile between these stages of the filtration, we will obtain a complicated expression for zero.

An illustration of the profiles for $\widetilde{G}_{\bullet}$ and $\tilde{X}_{\bullet}$ in Example 1 with $u=3, v=5$ is included below.

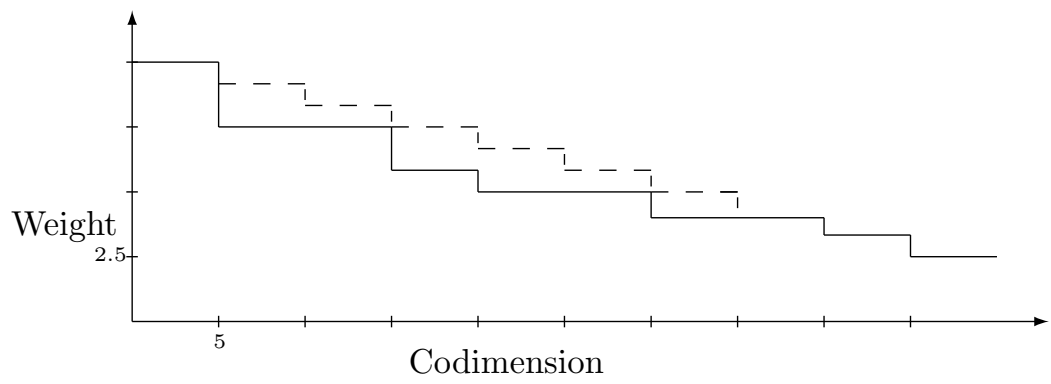

Figure 2. Profiles for $\widetilde{G}_{\bullet}$ (dashed) and $\widetilde{X} \bullet$ (solid) in Example 1 with $u=3, v=5$

4.2.2. Using the profile of $\widetilde{X}_{\bullet}$. Note that the spaces used to construct each $\widetilde{X}_{k, w}$ in Definition 4.1 satisfy the degree hypothesis of Lemma 3.2 every space going into the span is either of the form $\left(V_{k}^{u-w} V_{k+1}^{w} V_{0}\right)^{v}$ or $\left(V_{s(k, i)}^{u-W(k, w, i)} V_{t(k, i)}^{W(k, w, i)} V_{0}\right)^{v}$. But the base locus of any space of this form is bounded by the base locus of $\left(V_{N}^{u} V_{0}\right)^{v}$, which is $u v c_{\bar{N}, 1}+\cdots+u v c_{\bar{N}, q}$. That is, $\max _{j}\left\{d_{j, i}\right\} \leq u v c_{\bar{N}, i}$, so we have

$$
\sum_{i=1}^{q} \max _{j}\left\{d_{j, i}\right\} \leq \sum_{i=1}^{q} u v c_{\bar{N}, i} \leq u v d<u v d+u d-2 g=d m-2 g .
$$


Fix $(k, w)$ for this paragraph. If we knew that the space $\left(V_{k}^{u-w} V_{k+1}^{w} V_{0}\right)^{v}$ or $\left(V_{s(k, i)}^{u-W} V_{t(k, i)}^{W} V_{0}\right)^{v}$ appearing in $\widetilde{X}_{(k, w)}$ always minimized the multiplicity of $Q_{i}$ among these spaces appearing in $\widetilde{X}_{(k, w)}$, then we could apply Lemma 3.2 to conclude that $\widetilde{x}(k, w)=\sum_{i=1}^{q} \widetilde{x}(k, w, i)$. However, it is not true that $\left(V_{k}^{u-w} V_{k+1}^{w} V_{0}\right)^{v}$ or $\left(V_{s(k, i)}^{u-W} V_{t(k, i)}^{W} V_{0}\right)^{v}$ always minimizes the multiplicity of $Q_{i}$ among the spaces appearing in $\widetilde{X}_{(k, w)}$. (It is possible to find the minimum; see [Swin, pp. 63-64.) However, since the minimum multiplicity for the point $Q_{i}$ can only be smaller than the multiplicity $\widetilde{x}(k, w, i)$, we may conclude that $\widetilde{x}(k, w) \leq \sum_{i=1}^{q} \widetilde{x}(k, w, i)$. Of course, this is not enough to bound $\widetilde{x}(k, w+1)-\widetilde{x}(k, w)$. But since the $\widetilde{r}_{k}$ 's are decreasing, the weight $A$ of this profile will only decrease if some $\widetilde{x}(k, w)<\sum_{i=1}^{q} \widetilde{x}(k, w, i)$. So computing using equality at every stage gives the following upper bound for $A$ :

$$
A \leq \sum_{k=0}^{\widetilde{N}-1} \sum_{w=0}^{u-1}\left(v(u-w) r_{k}+v w r_{k+1}+v r_{0}\right)(\widetilde{x}(k, w+1)-\widetilde{x}(k, w))+\left(\operatorname{dim} \widetilde{X}_{\widetilde{N}}\right) v r_{0} .
$$

We have $\widetilde{X}_{\widetilde{N}}=H^{0}\left(C, \mathcal{O}(m)\left(-u v D_{\bar{N}}\right)\right)$, and so we may compute

$$
\operatorname{dim} \widetilde{X}_{\widetilde{N}}=d m-u v d_{\bar{N}}-g+1=\left(d-d_{\bar{N}}\right) u v+d v-g+1 .
$$

Substituting this into (13), we obtain

$$
\begin{aligned}
A & \leq \sum_{k=0}^{\widetilde{N}-1} \sum_{w=0}^{u-1}\left(v(u-w) r_{k}+v w r_{k+1}+v r_{0}\right)(\widetilde{x}(k, w+1)-\widetilde{x}(k, w)) \\
& +\left(\left(d-d_{\bar{N}}\right) u v+d v-g+1\right) v r_{0} .
\end{aligned}
$$

Rather than trying to bound the right hand side of (14), we will follow a different approach. We will define a "virtual" profile whose graph has area $A^{\text {vir }}$ nearly the same as the area of the graph $A$ of the actual profile, but which is computationally a little easier to work with. Let $\Delta=A-A^{\text {vir }}$ be the discrepancy. Also, for each $i$ between 1 and $q$, recall that $r_{j(i, 0)}$ is the $r_{j}$ such that $c_{j, i}=0$ and $c_{j+1, i}>0$. Then

$$
T \leq A^{\mathrm{vir}}+\Delta+\sum_{i=1}^{n} B_{i} r_{j(i, 0)}(u+1)^{2} v^{2} .
$$

We use the rest of this section to define the virtual profile. In the next section we bound $\Delta$, and in Section [6] we bound $A^{\mathrm{vir}}+\sum_{i=1}^{n} B_{i} r_{j(i, 0)}(u+1)^{2} v^{2}$. Putting this all together with (15), we will get a bound for $T$.

4.3. The virtual profile. The virtual profile simplifies the graph of the profile in three ways:

- In the profile, we form a span of $q$ spaces for all $k$ and for all $w$, so the step function is defined over $(\widetilde{N} \times u)+1$ intervals. In the virtual profile, we only partition the domain (the codimension axis) into $\widetilde{N}+1$ intervals.

- In the profile, we round so that $W=W(u, v ; k, w, i)$ is always an integer, so exponents, multiplicities, and codimensions are integers. In the virtual profile, their counterparts are rational numbers. (This is the origin of the adjective "virtual".)

- In particular, the quantity $\widetilde{f}(k)$ (defined below) is the virtual counterpart to $\widetilde{x}(k, 0)$. The profile is a step function, so the two points $\left(\widetilde{x}(k, 0), u v r_{k}+v r_{0}\right)$ and $\left(\widetilde{x}(k+1,0), u v r_{k+1}+v r_{0}\right)$ are connected by a staircase. But in the 
virtual profile, we connect the two points $\left(\widetilde{f}(k), \widetilde{r}_{k}\right)$ and $\left(\widetilde{f}(k+1), \widetilde{r}_{k+1}\right)$ by a straight line segment.

We will call the figure so obtained the virtual profile and use $A^{\text {vir }}$, the area under the virtual profile, to approximate $A$.

Definition 4.2 (The virtual profile). For each $k=0, \ldots, \widetilde{N}-1$, we define $\widetilde{f}(k)$ as follows. We begin by defining $\widetilde{f}_{i}(k)$ for each $i$. Fix $i$. Graph the set of points $\left\{\left(\widetilde{r}_{k(i, \ell)}, \widetilde{c}_{k(i, \ell)}\right): \ell=0, \ldots, K_{i}\right\}$ and connect these by straight line segments. Then $\widetilde{f}_{i}(k)$ is the piecewise linear function whose value at $k$ is the second coordinate of the point on this graph lying over $\widetilde{r}_{k}$.

The picture described above translates into the following rules. We refer to Cases I-IV as defined in 4.1 .3 .

- If $\widetilde{c}_{k+1, i}=0$, then $\widetilde{f}_{i}(k)=0$.

- Otherwise, let $s(k, i)$ and $t(k, i)$ be as defined at the beginning of Section 4.1.3, Then

$$
\widetilde{f}_{i}(k)=\left(\frac{\widetilde{r}_{k}-\widetilde{r}_{t(k, i)}}{\widetilde{r}_{s(k, i)}-\widetilde{r}_{t(k, i)}} \widetilde{c}_{s(k, i)}+\left(1-\frac{\widetilde{r}_{k}-\widetilde{r}_{t(k, i)}}{\widetilde{r}_{s(k, i)}-\widetilde{r}_{t(k, i)}}\right) \widetilde{c}_{t(k, i)}\right) .
$$

Note that in Case I and Case IV the formula above just gives $\widetilde{f}_{i}(k)=\widetilde{c}_{k, i}$, since $s(k, i)=k$ in these cases.

Finally,

$$
\widetilde{f}(k):=\sum_{i=1}^{q} \widetilde{f}_{i}(k) .
$$

The virtual profile is the graph of the piecewise linear function connecting the points $\left\{\left(\widetilde{f}(k), \widetilde{r}_{k}\right)\right\}$.

Note the switch in the order of the coordinates that takes place: $\widetilde{f}_{i}(k)$ is defined by a graph in the (weight $\times$ multiplicity of $Q_{i}$ )-plane, whereas the virtual profile is graphed along with the profile in the (codimension $\times$ weight)-plane.

In some examples, the vertices and line segments of the virtual profile have obvious algebro-geometric interpretations, but this is not true for all 1-PS. Therefore, it seems best to regard the virtual profile merely as a graph and not an algebrogeometric object of any kind.

The quantity $\widetilde{f}(k)$ is an approximate upper bound for the codimension of the $\widetilde{r}_{k}$-weight space in $H^{0}\left(C, \mathcal{O}_{C}(m)\right)$. We have

$$
\begin{aligned}
A^{\mathrm{vir}} & =\sum_{k=0}^{\widetilde{N}-1} \frac{1}{2}(\widetilde{f}(k+1)-\widetilde{f}(k))\left(\widetilde{r}_{k+1}+\widetilde{r}_{k}\right)+\left(\operatorname{dim} \widetilde{V}_{\widetilde{N}}\right) v r_{0} \\
& =\sum_{k=0}^{\widetilde{N}-1} \frac{1}{2}(\widetilde{f}(k+1)-\widetilde{f}(k))\left(\widetilde{r}_{k+1}+\widetilde{r}_{k}\right)+\left(d-d_{\bar{N}}\right) u v+d v-g+1 .
\end{aligned}
$$

Also, for each $i$ between 1 and $q$, recall that $r_{j(i, 0)}$ is the $r_{j}$ such that $c_{j, i}=0$ and $c_{j+1, i}>0$. Let $T^{\mathrm{vir}}=A^{\mathrm{vir}}+(u+1)^{2} v^{2} \sum_{i=1}^{q} B_{i} r_{j(i, 0)}$ denote the approximation to $T$ obtained by approximating $A$ by $A^{\text {vir }}$. We have the following upper bound for 
$T^{\mathrm{vir}}$ :

$$
\begin{aligned}
T^{\mathrm{vir}} \leq & \sum_{k=0}^{\widetilde{N}-1} \frac{1}{2}(\widetilde{f}(k+1)-\widetilde{f}(k))\left(\widetilde{r}_{k+1}+\widetilde{r}_{k}\right) \\
& +\left(\left(d-d_{\bar{N}}\right) u v+d v-g+1\right) v r_{0}+(u+1)^{2} v^{2} \sum_{i=1}^{n} B_{i} r_{j(i, 0)}
\end{aligned}
$$

4.3.1. Illustration: The virtual profile for $\widetilde{X}_{\bullet}$ in Example 1. Let us compute the virtual profile for Example 1. We can compute the virtual profile for an arbitrary $u, v$ :

For $k=0$ there is nothing to compute.

For $k=1$, the multiplicity of $P_{1}$ does not jump from row 1 to row 2 . We are in Case II. Looking at where the multiplicity $P_{1}$ jumps, we have $s(1,1)=0$ and $t(1,1)=3$, and we find that $\widetilde{f}_{1}(1)=\frac{1}{3} u v$. The multiplicity of $P_{2}$ jumps between row 1 and row 2 ; we are in Case IV, and we have $\widetilde{f}_{2}(1)=\widetilde{c}_{1,2}=0$. Finally, since the multiplicity of $P_{3}$ is zero in both row 1 and row $2, \widetilde{f}_{3}(1)=0$. Then $\widetilde{f}(1)=\frac{1}{3} u v$. Also, $\widetilde{r}_{1}=\frac{1}{3} u v+\frac{1}{2} v$.

For $k=2$, the multiplicity of $P_{1}$ does not jump from row 2 to row 3 . We are in Case II, $s(2,1)=0$ and $t(2,1)=3$, and $\widetilde{f}_{1}(2)=\frac{2}{3} u v$. The multiplicity of $P_{2}$ does not jump between row 2 and row 3 ; we are in Case II, and $s(2,2)=1$ and $t(2,2)=3$, giving $\widetilde{f}_{2}(2)=\frac{1}{2} u v$. Finally, the multiplicity of $P_{3}$ jumps at row 2 ; we are in Case IV, so $\widetilde{f}_{3}(2)=\widetilde{c}_{2,3}=0$. Then $\widetilde{f}(2)=\frac{7}{6} u v$. Also, $\widetilde{r}_{2}=\frac{1}{6} u v+\frac{1}{2} v$.

Finally, for $k=\widetilde{N}=3$ there is also nothing to compute.

The area of the region under the graph connecting the points $\left(0 u v, \frac{1}{2} u v+\frac{1}{2} v\right)$, $\left(\frac{1}{3} u v, \frac{1}{3} u v+\frac{1}{2} v\right),\left(\frac{7}{6} u v, \frac{1}{6} u v+\frac{1}{2} v\right)$ and $\left(3 u v, \frac{1}{2} v\right)$ is $\frac{1}{2} u^{2} v^{2}+\frac{3}{2} u v^{2}$. To this we add the weight of the $v r_{0}$ region, which is $\left(\operatorname{dim} \widetilde{V}_{\widetilde{N}}\right) v r_{0}=((d-3) u v+d v-g+1)\left(\frac{1}{2} v\right)$. We have

$$
A^{\mathrm{vir}}=\frac{1}{2} u^{2} v^{2}+\frac{1}{2} d u v^{2}+\frac{1}{2} d v^{2}-\frac{1}{2}(g-1) v .
$$

Using $B_{i}=\frac{1}{2}$, the contribution from the marked points is $\frac{1}{2}\left(u^{2} v^{2}+2 u v^{2}+v^{2}\right)$. We have

$$
T^{\mathrm{vir}}=1 u^{2} v^{2}+\left(\frac{1}{2} d+1\right) u v^{2}+\left(\frac{1}{2} d+1\right) v^{2}-\frac{1}{2}(g-1) v .
$$

4.3.2. The profile and virtual profile for Example 1 together. Here are the profile and virtual profile for $\widetilde{X}_{\bullet}$ in Example 1 with $u=3, v=5$.

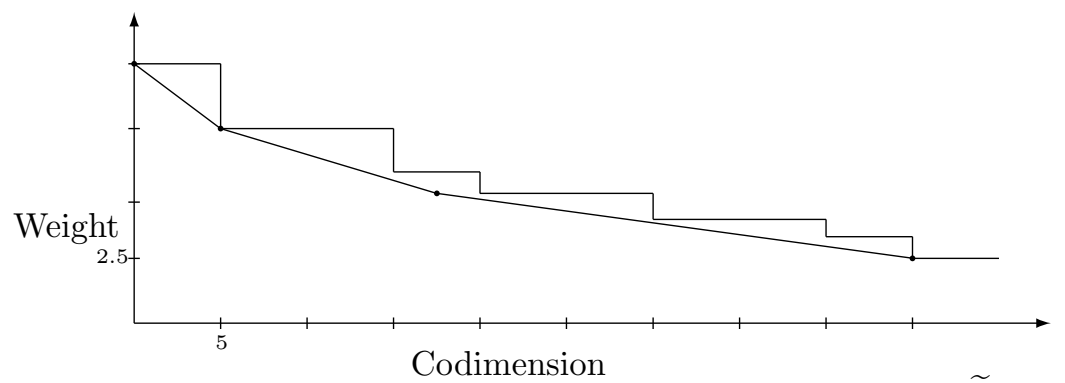

Figure 3. Example 1. Profile and virtual profile for $\tilde{X}_{\bullet}$ with $u=3, v=5$ 
In this picture the area under the profile looks significantly larger than the area under the virtual profile, but for larger values of $u$ the difference of these areas becomes relatively smaller. This is made rigorous in the next section, but as an example, an approximate picture of the profile and virtual profile for $\widetilde{X}_{\bullet}$ in Example 1 with $u=20, v=5$ is displayed in the introduction to this paper.

\section{The Discrepancy Between the Profile and virtual Profile for $\widetilde{X}$.}

In this section we bound the discrepancy $\Delta:=A-A^{\text {vir }}$ by computing bounds for several terms which contribute to it.

The area under the graph of the virtual profile may be divided in an obvious way into $\widetilde{N}$ vertical trapezoids and one final rectangle. Let us focus on the area $A_{k}^{\text {vir }}$ of the $k^{\text {th }}$ trapezoid:

$$
\begin{aligned}
A_{k}^{\mathrm{vir}} & =\frac{1}{2}(\widetilde{f}(k+1)-\widetilde{f}(k))\left(\widetilde{r}_{k+1}+\widetilde{r}_{k}\right) \\
& =\frac{1}{2}\left(\sum_{i=1}^{q} \widetilde{f}_{i}(k+1)-\sum_{i=1}^{q} \widetilde{f}_{i}(k)\right)\left(\widetilde{r}_{k+1}+\widetilde{r}_{k}\right) \\
& =\sum_{i=1}^{q} \frac{1}{2}\left(\widetilde{f}_{i}(k+1)-\widetilde{f}_{i}(k)\right)\left(\widetilde{r}_{k+1}+\widetilde{r}_{k}\right) .
\end{aligned}
$$

We will write $A_{k, i}^{\mathrm{vir}}$ for the $i^{\text {th }}$ summand:

$$
A_{k, i}^{\mathrm{vir}}=\frac{1}{2}\left(\widetilde{f}_{i}(k+1)-\widetilde{f}_{i}(k)\right)\left(\widetilde{r}_{k+1}+\widetilde{r}_{k}\right)
$$

We now compute $A_{k, i}^{\mathrm{vir}}$.

5.1. Computing $A_{k, i}^{\mathrm{vir}} . A_{k, i}^{\mathrm{vir}}$ is the area of the trapezoid whose vertices are $\left(\widetilde{f}_{i}(k), 0\right)$, $\left(\widetilde{f}_{i}(k+1), 0\right),\left(\widetilde{f}_{i}(k+1), \widetilde{r}_{k+1}\right)$, and $\left(\widetilde{f}_{i}(k), \widetilde{r}_{k}\right)$. To compute $\widetilde{f}_{i}(k+1)-\widetilde{f}_{i}(k)$, recall the definition of $\tilde{f}_{i}(k)$ given in Definition 4.2 and the four cases defined in Section 4.1 .3 .

We do the calculation for Case II. Recall that Case II occurs when the multiplicity $\widetilde{c}_{\bullet}, i$ does not jump at row $k$ or at row $k+1$, and as a result, $(s(k, i), t(k, i))=$ $(s(k+1, i), t(k+1, i))$. In the profile, spaces of the form $V_{s(k, i)}^{(u-W) v} V_{t(k, i)}^{W v} V_{0}^{v}$ are being used between these two vertices. In the virtual profile, we are calculating as if spaces of the form $V_{s(k, i)}^{\alpha u v} V_{t(k, i)}^{(1-\alpha) v} V_{0}^{v}$ were being used between these two vertices (where $\alpha$ ranges over a subinterval strictly in the interior of $[0,1]$ ).

We have:

$$
\tilde{f}_{i}(k+1)=\frac{\widetilde{r}_{k+1}-\widetilde{r}_{t(k+1, i)}}{\widetilde{r}_{s(k+1, i)}-\widetilde{r}_{t(k+1, i)}} \widetilde{c}_{s(k+1, i)}+\left(1-\frac{\widetilde{r}_{k+1}-\widetilde{r}_{t(k+1, i)}}{\widetilde{r}_{s(k+1, i)}-\widetilde{r}_{t(k+1, i)}}\right) \widetilde{c}_{t(k+1, i)}
$$

and

$$
\widetilde{f}_{i}(k)=\frac{\widetilde{r}_{k}-\widetilde{r}_{t(k, i)}}{\widetilde{r}_{s(k, i)}-\widetilde{r}_{t(k, i)}} \widetilde{c}_{s(k, i)}+\left(1-\frac{\widetilde{r}_{k}-\widetilde{r}_{t(k, i)}}{\widetilde{r}_{s(k, i)}-\widetilde{r}_{t(k, i)}}\right) \widetilde{c}_{t(k, i)}
$$


and $(s(k, i), t(k, i))=(s(k+1, i), t(k+1, i))$. Thus

$$
\begin{aligned}
A_{k, i}^{\mathrm{vir}}= & \frac{1}{2}\left(\widetilde{r}_{k+1}+\widetilde{r}_{k}\right)\left(\widetilde{f}_{i}(k+1)-\widetilde{f}_{i}(k)\right) \\
= & \frac{1}{2}\left(\widetilde{r}_{k+1}+\widetilde{r}_{k}\right)\left(\frac{\widetilde{r}_{k}-\widetilde{r}_{k+1}}{\widetilde{r}_{s(k, i)}-\widetilde{r}_{t(k, i)}}\left(\widetilde{c}_{t(k, i)}-\widetilde{c}_{s(k, i)}\right)\right) \\
= & \frac{1}{2}\left(u v r_{k+1}+u v r_{k}+2 v r_{0}\right)\left(u v \frac{r_{k}-r_{k+1}}{r_{s(k, i)}-r_{t(k, i)}}\left(c_{t(k, i)}-c_{s(k, i)}\right)\right) \\
= & u^{2} v^{2}\left(\frac{1}{2}\left(r_{k+1}+r_{k}\right)\left(c_{t(k, i)}-c_{s(k, i)}\right) \frac{r_{k}-r_{k+1}}{r_{s(k, i)}-r_{t(k, i)}}\right) \\
& \quad+u v^{2}\left(r_{0}\left(c_{t(k, i)}-c_{s(k, i)}\right) \frac{r_{k}-r_{k+1}}{r_{s(k, i)}-r_{t(k, i)}}\right) .
\end{aligned}
$$

By similar calculations, and using some of the information presented in paragraphs I, III and IV above, we derive the same formula in Cases I, III and IV.

5.2. Computing bounds for $A_{k, i}$. We have defined $A_{k, i}^{\mathrm{vir}}$ but have not yet defined a corresponding quantity $A_{k, i}$. We do this now. Let $A_{k, i}$ denote the following sum:

$$
A_{k, i}:=\sum_{w=0}^{u-1}\left((u-w) r_{k}+w r_{k+1}+r_{0}\right)(\widetilde{x}(k, w+1, i)-\widetilde{x}(k, w, i)) .
$$

In pictures, $\sum_{i=1}^{q} A_{k, i}$ is the area under the profile between $\widetilde{x}(k, 0)$ and $\widetilde{x}(k+1,0)$.

We wish to bound $A_{k, i}$. We split into Cases I-IV as in Section 5.1. From here to the end of Section 5.2, we suppress the subscripts $k, i$ as much as possible, as they do not change. We reintroduce them at the end of this subsection in (30).

Recall that in Definition 4.1, for each $w$, we defined $W=W(k, w, i)$ to be the smallest integer such that the space $\left(V_{s}^{u-W} V_{t}^{W} V_{0}\right)^{v}$ has weight less than or equal to $v(u-w) r_{k}+v w r_{k+1}+v r_{0}$. We use this property to get an expression for $W$ :

$$
\begin{aligned}
v(u-W) r_{s}+v W r_{t}+v r_{0} & \leq v(u-w) r_{k}+v w r_{k+1}+v r_{0} \\
\Longleftrightarrow W & \geq \frac{u\left(r_{s}-r_{k}\right)+w\left(r_{k}-r_{k+1}\right)}{r_{s}-r_{t}} \\
\Longrightarrow W(w)=W(k, w, i) & =\left\lceil\frac{u\left(r_{s}-r_{k}\right)+w\left(r_{k}-r_{k+1}\right)}{r_{s}-r_{t}}\right\rceil .
\end{aligned}
$$

It is useful to write

$$
\begin{aligned}
\zeta=\zeta_{k, i} & :=\frac{r_{k}-r_{k+1}}{r_{s}-r_{t}}, \\
\xi=\xi_{k, i} & :=\frac{r_{s}-r_{k}}{r_{s}-r_{t}} .
\end{aligned}
$$

Then

$$
W=\lceil u \xi+w \zeta\rceil
$$

Since $s \leq k$ and $k \leq t$ and $s<t$, we have $0 \leq \zeta \leq 1$ and $0 \leq \xi \leq 1$. 
Proceeding, we have:

$$
\begin{aligned}
\widetilde{x}(k, w, i) & =v(u-W(w)) c_{s}+v W(w) c_{t}, \\
\widetilde{x}(k, w+1, i) & =v(u-W(w+1)) c_{s}+v W(w+1) c_{t} \\
\Longrightarrow \widetilde{x}(k, w+1, i)-\widetilde{x}(k, w, i) & =v\left(c_{t}-c_{s}\right)(W(w+1)-W(w)) .
\end{aligned}
$$

Putting this into (19) we have:

$$
\begin{aligned}
A_{k, i}= & \sum_{w=0}^{u-1} v\left((u-w) r_{k}+w r_{k+1}+r_{0}\right) v\left(\left(c_{t}-c_{s}\right)(W(w+1)-W(w))\right) \\
= & v^{2}\left(c_{t}-c_{s}\right)\left(\sum_{w=0}^{u-1}\left(u r_{k}+r_{0}-w\left(r_{k}-r_{k+1}\right)\right)(W(w+1)-W(w))\right) \\
= & v^{2}\left(c_{t}-c_{s}\right)\left(\left(u r_{k}+r_{0}\right) \sum_{w=0}^{u-1}(W(w+1)-W(w))\right. \\
& \left.-\left(r_{k}-r_{k+1}\right) \sum_{w=0}^{u-1} w(W(w+1)-W(w))\right) .
\end{aligned}
$$

We consider the sums in (24). Use the notation

$$
\{y\}:=y-\lfloor y\rfloor
$$

for the fractional part of $y$. Define

$$
\eta:=\left\{\begin{array}{l}
\{u \xi\}-1, \text { if }\{u \zeta+\{u \xi\}-1\}=0 \\
\{u \xi\}-\{u \zeta+\{u \xi\}-1\}, \text { if }\{u \zeta+\{u \xi\}-1\} \neq 0 .
\end{array}\right.
$$

Note that $-1 \leq \eta<1$.

Finally, let $\mathbf{1}_{\zeta \neq 0}$ and $\mathbf{1}_{\{u \xi\}=0}$ be indicator functions. Then the sums in (24) are as follows:

Lemma 5.1 (Swin Lemma 2.6.1).

$$
\begin{aligned}
\sum_{w=0}^{u-1} w(W(w+1)-W(w)) & =\mathbf{1}_{\zeta \neq 0} \sum_{\ell=1}^{u \zeta+\eta}\left\lfloor\frac{\ell-\{u \xi\}}{\zeta}\right\rfloor \\
\sum_{w=0}^{u-1}(W(w+1)-W(w)) & =u \zeta+\eta+\mathbf{1}_{\{u \xi\}=0} .
\end{aligned}
$$


We substitute in (28) and (27) into (24) and proceed:

$$
\begin{aligned}
& A_{k, i}=v^{2}\left(c_{t}-c_{s}\right)\left(\left(u r_{k}+r_{0}\right)\left(u \zeta+\eta+\mathbf{1}_{\{u \xi\}=0}\right)\right. \\
& \left.-\left(r_{k}-r_{k+1}\right) \mathbf{1}_{\zeta \neq 0} \sum_{\ell=1}^{u \zeta+\eta}\left\lfloor\frac{\ell-\{u \xi\}}{\zeta}\right\rfloor\right) \\
& =v^{2}\left(c_{t}-c_{s}\right)\left(\left(u r_{k}+r_{0}\right)\left(u \zeta+\eta+\mathbf{1}_{\{u \xi\}=0}\right)\right. \\
& \left.-\left(r_{k}-r_{k+1}\right) \mathbf{1}_{\zeta \neq 0} \sum_{\ell=1}^{u \zeta+\eta}\left(\frac{\ell}{\zeta}-\frac{\{u \xi\}}{\zeta}-\left\{\frac{\ell-\{u \xi\}}{\zeta}\right\}\right)\right) \\
& =v^{2}\left(c_{t}-c_{s}\right)\left(u^{2} r_{k} \zeta+\left(\left(\eta+\mathbf{1}_{\{u \xi\}=0}\right) r_{k}+\zeta r_{0}\right) u+r_{0}\left(\eta+\mathbf{1}_{\{u \xi\}=0}\right)\right. \\
& -\mathbf{1}_{\zeta \neq 0} \frac{\left(r_{k}-r_{k+1}\right)}{\zeta}\left(\frac{1}{2}(u \zeta+\eta)(u \zeta+\eta+1)\right) \\
& \left.+\mathbf{1}_{\zeta \neq 0} \frac{\left(r_{k}-r_{k+1}\right)}{\zeta} \sum_{\ell=1}^{u \zeta+\eta}\left(\{u \xi\}+\zeta\left\{\frac{\ell-\{u \xi\}}{\zeta}\right\}\right)\right) \text {. }
\end{aligned}
$$

In the last line, we have $0 \leq\left\{\frac{\ell-\{u \xi\}}{\zeta}\right\}<1$. We apply this bound to obtain an upper bound for $A_{k, i}$. We also group terms by their $u$-degree and restore the $k, i$ symbols which have been suppressed throughout this subsection, yielding:

$$
\left.\begin{array}{rl}
A_{k, i} \leq & v^{2}\left(c_{t(k, i)}-c_{s(k, i)}\right)\left(\left(\frac{1}{2}\left(r_{k}+r_{k+1}\right) \zeta_{k, i}\right) u^{2}\right. \\
+ & \left(\left(\eta_{k, i}+\mathbf{1}_{\left\{u \xi_{k, i}\right\}=0}\right) r_{k}+\zeta_{k, i} r_{0}+\mathbf{1}_{\zeta_{k, i} \neq 0}\left(r_{k}-r_{k+1}\right)\right. \\
& \left.\times\left(\left\{u \xi_{k, i}\right\}+\zeta_{k, i}-\eta_{k, i}-\frac{1}{2}\right)\right) u \\
+ & \left(r_{0}\left(\eta_{k, i}+\mathbf{1}_{\left\{u \xi_{k, i}\right\}=0}\right)+\mathbf{1}_{\zeta_{k, i} \neq 0} \frac{\left(r_{k}-r_{k+1}\right)}{\zeta_{k, i}}\right. \\
\left.\times\left(\eta_{k, i}\left\{u \xi_{k, i}\right\}+\zeta_{k, i} \eta_{k, i}-\frac{1}{2} \eta_{k, i}\left(\eta_{k, i}+1\right)\right)\right) 1
\end{array}\right) .
$$

This completes our calculation of $A_{k, i}$ in the general case. 
5.3. Bounding the discrepancy. We now have all the ingredients we need to bound $\Delta$ :

$$
\Delta:=A-A^{\mathrm{vir}} \leq \sum_{k=0}^{\widetilde{N}-1} \sum_{i=1}^{q}\left(A_{k, i}-A_{k, i}^{\mathrm{vir}}\right) .
$$

By comparing (18) and (30) and by using the definition of $\zeta_{k, i}$ at (21), we see that

$$
\begin{aligned}
& \Delta_{k, i}:=A_{k, i}-A_{k, i}^{\mathrm{vir}} \\
& \leq v^{2}\left(c_{t(k, i)}-c_{s(k, i)}\right)\left(\left(\left(\eta_{k, i}+\mathbf{1}_{\left\{u \xi_{k, i}\right\}=0}\right) r_{k}+\mathbf{1}_{\zeta_{k, i} \neq 0}\left(r_{k}-r_{k+1}\right)\right.\right. \\
& \left.\times\left(\left\{u \xi_{k, i}\right\}+\zeta_{k, i}-\eta_{k, i}-\frac{1}{2}\right)\right) u \\
& +\left(\left(\eta_{k, i}+\mathbf{1}_{\left\{u \xi_{k, i}\right\}=0}\right) r_{0}+\mathbf{1}_{\zeta_{k, i} \neq 0}\left(r_{s(k, i)}-r_{t(k, i)}\right)\right. \\
& \left.\left.\times\left(\eta_{k, i}\left\{u \xi_{k, i}\right\}+\zeta_{k, i} \eta_{k, i}-\frac{1}{2} \eta_{k, i}^{2}-\frac{1}{2} \eta_{k, i}\right)\right) 1\right) .
\end{aligned}
$$

Recall that the weights $r_{j}$ and the fractional parts of any quantity must be between 0 and 1 , and $-1 \leq \eta_{k, i}<1$. Therefore we may make various coarse estimates:

$$
\begin{aligned}
\left(\eta_{k, i}+\mathbf{1}_{\left\{u \xi_{k, i}\right\}=0}\right) r_{k} & <2, \\
\left\{u \xi_{k, i}\right\}+\zeta_{k, i}-\eta_{k, i}-\frac{1}{2} & <1+1+1-\frac{1}{2} \\
\Rightarrow \mathbf{1}_{\zeta_{k, i} \neq 0}\left(r_{k}-r_{k+1}\right)\left(\left\{u \xi_{k, i}\right\}+\zeta_{k, i}-\eta_{k, i}-\frac{1}{2}\right) & <\frac{5}{2}, \\
\left(\eta_{k, i}+\mathbf{1}_{\left\{u \xi_{k, i}\right\}=0}\right) r_{0} & <2, \\
\eta_{k, i}\left\{u \xi_{k, i}\right\}+\zeta_{k, i} \eta_{k, i}-\frac{1}{2} \eta_{k, i}^{2}-\frac{1}{2} \eta_{k, i} & <1+1-0+\frac{1}{2} \\
(32) \Rightarrow \mathbf{1}_{\zeta_{k, i} \neq 0}\left(r_{s}-r_{t}\right)\left(\eta_{k, i}\left\{u \xi_{k, i}\right\}+\zeta_{k, i} \eta_{k, i}-\frac{1}{2} \eta_{k, i}^{2}-\frac{1}{2} \eta_{k, i}\right) & <\frac{5}{2} .
\end{aligned}
$$

Combining these inequalities with (31) we obtain:

$$
\Delta_{k, i} \leq u v^{2}\left(c_{t(k, i)}-c_{s(k, i)}\right)\left(\frac{9}{2}\right)+v^{2}\left(c_{t(k, i)}-c_{s(k, i)}\right)\left(\frac{9}{2}\right) .
$$

Next, we claim that the estimates given for each $k$ in (33) together yield

$$
\sum_{k=0}^{\widetilde{N}-1} \Delta_{k, i} \leq u v^{2}\left(\frac{9}{2} c_{\bar{N}, i}\right)+v^{2}\left(\frac{9}{2} c_{\bar{N}, i}\right) .
$$

Refer back to the definition of $s$ and $t$ in Section 4.1. Equation (34) follows because the pairs $(s, t)$ fit together in such a way that when the estimates from (33) are summed over $k$, the sum telescopes. 
Finally, using the estimates obtained in (34), we obtain

$$
\Delta \leq \sum_{i=1}^{q} \sum_{k=0}^{\widetilde{N}-1} \Delta_{k, i} \leq u v^{2}\left(\frac{9}{2} d\right)+v^{2}\left(\frac{9}{2} d\right) .
$$

Observe that $\Delta$ is of order $u v^{2}$ and not of order $u^{2} v^{2}$.

\section{Bounding the Weight of the VIRTuAL PRofile $T^{\text {vir }}$}

We use the subscript notation introduced in Section 4.1 .

6.1. Preparing for comparison. Recall that in (17) we obtained the following bound on $T^{\mathrm{vir}}$ :

$$
\begin{aligned}
T^{\mathrm{vir}} \leq \sum_{k=0}^{\tilde{N}-1} \frac{1}{2}(\tilde{f}(k+1)-\tilde{f}(k))\left(\widetilde{r}_{k+1}+\widetilde{r}_{k}\right)+\left(\left(d-d_{\bar{N}}\right) u v+d v-g+1\right) v r_{0} \\
\quad+(u+1)^{2} v^{2} \sum_{i=1}^{q} B_{i} r_{j(i, 0)} \\
=\sum_{k=0}^{\widetilde{N}-1} \frac{1}{2}(\tilde{f}(k+1)-\tilde{f}(k))\left(\widetilde{r}_{k+1}+\widetilde{r}_{k}\right)+\left(\left(d-d_{\bar{N}}\right) u v+d v-g+1\right) v r_{0} \\
\quad+u^{2} v^{2} \sum_{i=1}^{q} B_{i} r_{j(i, 0)}+\sum_{i=1}^{q} B_{i} r_{j(i, 0)}\left(2 u v^{2}+v^{2}\right) .
\end{aligned}
$$

Everything in this sum is in terms of $k$ (it is, after all, the weight of a basis of $\left.H^{0}\left(C, \mathcal{O}_{C}(m)\right)\right)$. Almost the only bound available is that the weights sum to 1 : $\sum_{j=0}^{\bar{N}} z_{j} r_{j}=1$. Our goal in this subsection is to rewrite (36) in a form that makes it easy to compare to $\sum z_{j} r_{j}$.

We focus on the first term of (36):

$$
\begin{aligned}
\sum_{k=0}^{\widetilde{N}-1} \frac{1}{2}(\widetilde{f}(k+1)-\widetilde{f}(k))\left(\widetilde{r}_{k+1}+\widetilde{r}_{k}\right) & =\sum_{k=0}^{\widetilde{N}-1} \frac{1}{2}\left(\sum_{i=1}^{q} \widetilde{f}_{i}(k+1)-\sum_{i=1}^{q} \widetilde{f}_{i}(k)\right)\left(\widetilde{r}_{k+1}+\widetilde{r}_{k}\right) \\
& =\sum_{k=0}^{\widetilde{N}-1} \sum_{i=1}^{q} \frac{1}{2}\left(\widetilde{f}_{i}(k+1)-\widetilde{f}_{i}(k)\right)\left(\widetilde{r}_{k+1}+\widetilde{r}_{k}\right) .
\end{aligned}
$$

Let $A_{k, i}^{\mathrm{vir}}$ denote the area of the region described in Definition 4.2. Then we have:

$$
\sum_{k=0}^{\widetilde{N}-1} \sum_{i=1}^{q} \frac{1}{2}\left(\widetilde{f}_{i}(k+1)-\widetilde{f}_{i}(k)\right)\left(\widetilde{r}_{k+1}+\widetilde{r}_{k}\right)=\sum_{k=0}^{\widetilde{N}-1} \sum_{i=1}^{q} A_{k, i}^{\mathrm{vir}}=\sum_{i=1}^{q} \sum_{k=0}^{\widetilde{N}-1} A_{k, i}^{\mathrm{vir}},
$$

where on the right we have changed the order of summation. Let $A_{i}^{\mathrm{vir}}=\sum_{k=0}^{\widetilde{N}-1} A_{k, i}^{\mathrm{vir}}$. Observe that, for a fixed $i$, it may not be necessary to partition this region into $\widetilde{N}$ vertical trapezoids to compute the area $A_{i}^{\text {vir }}$. A partition corresponding to the domains of definition of the piecewise linear function $f_{i}$, which may be coarser than that given by the full set of $k$ 's, will do. 
Recall that $k(i, \bullet)$ indexes the rows $k$ where the multiplicity $\widetilde{c}_{\bullet}, i$ jumps. Then we may compute:

$$
\begin{aligned}
A_{i}^{\mathrm{vir}} & =\sum_{k=0}^{\tilde{N}-1} \frac{1}{2}\left(\widetilde{f}_{i}(k+1)-\widetilde{f}_{i}(k)\right)\left(\widetilde{r}_{k+1}+\widetilde{r}_{k}\right) \\
& =\sum_{\ell=0}^{K_{i}-1} \frac{1}{2}\left(\widetilde{c}_{k(i, \ell+1)}-\widetilde{c}_{k(i, \ell)}\right)\left(\widetilde{r}_{k(i, \ell+1)}+\widetilde{r}_{k(i, \ell)}\right) \\
& =u^{2} v^{2}\left(\sum_{\ell=0}^{K_{i}-1} \frac{1}{2}\left(c_{j(i, \ell+1)}-c_{j(i, \ell)}\right)\left(r_{j(i, \ell+1)}+r_{j(i, \ell)}\right)\right)+u v^{2}\left(c_{\bar{N}, i} r_{0}\right) .
\end{aligned}
$$

We develop the coefficient of the $u^{2} v^{2}$ term of (39):

$$
\begin{aligned}
& \left(\sum_{\ell=0}^{K_{i}-1} \frac{1}{2}\left(c_{j(i, \ell+1)}-c_{j(i, \ell)}\right)\left(r_{j(i, \ell+1)}+r_{j(i, \ell)}\right)\right) \\
& =\left(\sum_{\ell=1}^{K_{i}} \frac{1}{2}\left(c_{j(i, \ell)}-c_{j(i, \ell-1)}\right) r_{j(i, \ell)}+\sum_{\ell=0}^{K_{i}-1} \frac{1}{2}\left(c_{j(i, \ell+1)}-c_{j(i, \ell)}\right) r_{j(i, \ell)}\right) \\
& =\left(\sum_{\ell=1}^{K_{i}-1} \frac{1}{2}\left(c_{j(i, \ell+1)}-c_{j(i, \ell-1)}\right) r_{j(i, \ell)}+\frac{1}{2} c_{j(i, 1)} r_{j(i, 0)}\right) .
\end{aligned}
$$

Once again, $c_{j(i, 1)}$ is the first nonzero multiplicity of $Q_{i}$ in a base locus in $V_{\bullet}$, and $r_{j(i, 0)}$ is the least weight of a section not vanishing at $Q_{i}$. Putting (40), (39), (38) and (37) into (36), we have

$$
\begin{aligned}
T^{\mathrm{vir}} & \leq u^{2} v^{2} \sum_{i=1}^{q}\left(\sum_{\ell=1}^{K_{i}-1} \frac{1}{2}\left(c_{j(i, \ell+1)}-c_{j(i, \ell-1)}\right) r_{j(i, \ell)}+\left(\frac{1}{2} c_{j(i, 1)}+B_{i}\right) r_{j(i, 0)}\right) \\
& +\left(\sum_{i=1}^{q} B_{i} r_{j(i, 0)}\right)\left(2 u v^{2}+v^{2}\right)+\left(\left(d-d_{\bar{N}}\right) u v+d v-g+1\right) v r_{0} \\
& +u v^{2}\left(\sum_{i=1}^{q} c_{\bar{N}, i} r_{0}\right)
\end{aligned}
$$

It is convenient to define $\mathcal{I}_{j}$ to be the set of $i$ 's where the multiplicity jumps at row $j$, and not for the first or last time:

$$
\mathcal{I}_{j}:=\left\{i \mid \exists \ell \neq 0, K_{i} \text { s.t. } j=j(i, \ell)\right\} .
$$

We switch the order of summations in (41) to obtain:

$$
\begin{aligned}
T^{\mathrm{vir}} \leq & u^{2} v^{2} \sum_{j=0}^{\bar{N}}\left(\sum_{\mathcal{I}_{j}} \frac{1}{2}\left(c_{j(i, \ell+1)}-c_{j(i, \ell-1)}\right)+\sum_{i: j=j(i, 0)}\left(\frac{1}{2} c_{j(i, 1)}+B_{i}\right)\right) r_{j} \\
& +\left(\sum_{i=1}^{q} B_{i} r_{j(i, 0)}\right)\left(2 u v^{2}+v^{2}\right)+(d u v+d v-g+1) v r_{0}
\end{aligned}
$$

which is of the form we desired. 
6.2. Comparison. The next lemma gives a bound for the coefficient of $u^{2} v^{2}$ in (43).

\section{Lemma 6.1.}

$$
\sum_{j=0}^{\bar{N}}\left(\sum_{\mathcal{I}_{j}} \frac{1}{2}\left(c_{j(i, \ell+1)}-c_{j(i, \ell-1)}\right)+\sum_{i: j=j(i, 0)}\left(\frac{1}{2} c_{j(i, 1)}+B_{i}\right)\right) r_{j} \leq \sum_{j=0}^{\bar{N}} Z_{j} r_{j},
$$

where

$$
Z_{j}:=\left\{\begin{array}{cc}
z_{j}, & j<j_{R R}, \\
z_{j}+\left(\sum_{\tau=0}^{j} z_{\tau}-(N-g)\right), & j=j_{R R}, \\
2 z_{j}, & j \geq j_{\text {Cliff }} .
\end{array}\right.
$$

Proof. We may rewrite the desired inequality as

$$
\sum_{j=0}^{\bar{N}}\left(Z_{j}-\sum_{i: j=j(i, 0)}\left(\frac{1}{2} c_{j(i, 1)}+B_{i}\right)-\sum_{\mathcal{I}_{j}} \frac{1}{2}\left(c_{j(i, \ell+1)}-c_{j(i, \ell-1)}\right)\right) r_{j} \geq 0 .
$$

We work successively on each index $j$ where

$$
Z_{j}-\sum_{i: j=j(i, 0)}\left(\frac{1}{2} c_{j(i, 1)}+B_{i}\right)-\sum_{\mathcal{I}_{j}} \frac{1}{2}\left(c_{j(i, \ell+1)}-c_{j(i, \ell-1)}\right)<0 .
$$

If there are no such $j$, we are done. So suppose there is at least one such index, and let the set of these be indexed $j_{e}$ beginning with $e=1$. By the definition of $j_{1}$ we have

$$
Z_{j}-\sum_{i: j=j(i, 0)}\left(\frac{1}{2} c_{j(i, 1)}+B_{i}\right)-\sum_{\mathcal{I}_{j}} \frac{1}{2}\left(c_{j(i, \ell+1)}-c_{j(i, \ell-1)}\right)>0
$$

for all $j<j_{1}$, so

$$
\begin{aligned}
& \sum_{j=0}^{j_{1}-1}\left(Z_{j}-\sum_{i: j=j(i, 0)}\left(\frac{1}{2} c_{j(i, 1)}+B_{i}\right)-\sum_{\mathcal{I}_{j}} \frac{1}{2}\left(c_{j(i, \ell+1)}-c_{j(i, \ell-1)}\right)\right) r_{j} \\
& \geq \sum_{j=0}^{j_{1}-1}\left(Z_{j}-\sum_{i: j=j(i, 0)}\left(\frac{1}{2} c_{j(i, 1)}+B_{i}\right)-\sum_{\mathcal{I}_{j}} \frac{1}{2}\left(c_{j(i, \ell+1)}-c_{j(i, \ell-1)}\right)\right) r_{j_{1}}
\end{aligned}
$$

and

$$
\sum_{j=0}^{j_{1}-1}\left(Z_{j}-\sum_{i: j=j(i, 0)}\left(\frac{1}{2} c_{j(i, 1)}+B_{i}\right)-\sum_{\mathcal{I}_{j}} \frac{1}{2}\left(c_{j(i, \ell+1)}-c_{j(i, \ell-1)}\right)\right) \geq 0 .
$$

We wish to establish that

$$
\begin{aligned}
& \sum_{j=0}^{j_{1}}\left(Z_{j}-\sum_{i: j=j(i, 0)}\left(\frac{1}{2} c_{j(i, 1)}+B_{i}\right)-\sum_{\mathcal{I}_{j}} \frac{1}{2}\left(c_{j(i, \ell+1)}-c_{j(i, \ell-1)}\right)\right) r_{j} \\
& \geq \sum_{j=0}^{j_{1}}\left(Z_{j}-\sum_{i: j=j(i, 0)}\left(\frac{1}{2} c_{j(i, 1)}+B_{i}\right)-\sum_{\mathcal{I}_{j}} \frac{1}{2}\left(c_{j(i, \ell+1)}-c_{j(i, \ell-1)}\right)\right) r_{j_{1}}
\end{aligned}
$$


(which is easy) and that

$$
\sum_{j=0}^{j_{1}}\left(Z_{j}-\sum_{i: j=j(i, 0)}\left(\frac{1}{2} c_{j(i, 1)}+B_{i}\right)-\sum_{\mathcal{I}_{j}} \frac{1}{2}\left(c_{j(i, \ell+1)}-c_{j(i, \ell-1)}\right)\right) \geq 0 .
$$

We rewrite this last inequality as

$$
\left(\sum_{j=0}^{j_{1}} Z_{j}\right)-\sum_{j=0}^{j_{1}}\left(\sum_{i: j=j(i, 0)}\left(\frac{1}{2} c_{j(i, 1)}+B_{i}\right)+\sum_{\mathcal{I}_{j}} \frac{1}{2}\left(c_{j(i, \ell+1)}-c_{j(i, \ell-1)}\right)\right) \geq 0 .
$$

We study the second sum in (44) above. Each $i$ falls into exactly one of the following cases:

Case 0. If $c_{\bullet}, i$ does not jump before or at $j_{1}$-that is, $j(i, 0)>j_{1}$-then this $i$ does not contribute.

Case 1. If $c_{\bullet}, i$ jumps exactly once before or at $j_{1}$ - that is, $j(i, 0) \leq j_{1}<j(i, 1)$ then this $i$ contributes

$$
\frac{1}{2} c_{j(i, 1)}+B_{i} \leq \frac{1}{2} c_{j_{1}+1, i}+\frac{1}{2} \leq c_{j_{1}+1, i},
$$

since $c_{j(i, 1)}=c_{j_{1}+1, i}, B_{i} \leq \frac{1}{2}$ and $c_{j_{1}+1, i} \geq 1$.

Case 2. If $c_{\bullet}, i$ jumps exactly twice before or at $j_{1}$ - that is, $j(i, 1) \leq j_{1}<j(i, 2)$ then the contribution to the second term is

$$
\frac{1}{2} c_{j(i, 1)}+B_{i}+\frac{1}{2} c_{j(i, 2)} \leq c_{j_{1}+1, i} .
$$

This follows because $c_{j(i, 2)}=c_{j_{1}+1, i}$ and $c_{j(i, 2)} \geq c_{j(i, 1)}+1$.

Case 3. If $c_{\bullet}, i$ jumps three or more times before or at $j_{1}$, then some telescoping occurs, and the contribution is

$$
\frac{1}{2} c_{j(i, 1)}+B_{i}+\frac{1}{2} c_{t\left(j_{1}, i\right)}+\frac{1}{2} c_{s\left(j_{1}, i\right)}-\frac{1}{2} c_{j(i, 1)} \leq c_{j_{1}+1, i} .
$$

Here we are abusing notation a little (according to Section 4.1 the first argument of $s(\bullet, i)$ or $t(\bullet, i)$ is supposed to be a $k$, not a $j)$. Here $s\left(j_{1}, i\right)$ denotes the largest index less than or equal to $j_{1}$ where $c_{\bullet}, i$ jumps, and $t\left(j_{1}, i\right)$ denotes the smallest index strictly greater than $j_{1}$ index where $c_{\bullet}, i$ jumps. Thus, $c_{t\left(j_{1}, i\right)}=c_{j_{1}+1, i}$ and $c_{s\left(j_{1}, i\right)} \leq c_{j_{1}, i}$.

To summarize, in each of Cases 0-3 above we see that the contribution is no more than $c_{j_{1}+1, i}$.

If $j_{1}<j_{R R}$, so that $j_{1}+1$ is in the Riemann-Roch region, then by (5) we have

$$
\sum_{i=1}^{q} c_{j_{1}+1, i} \leq \sum_{j=0}^{j_{1}} z_{j}
$$

so the left hand side of (44) is indeed nonnegative:

$$
\begin{gathered}
\left(\sum_{j=0}^{j_{1}} Z_{j}\right)-\sum_{j=0}^{j_{1}}\left(\sum_{i: j=j(i, 0)}\left(\frac{1}{2} c_{j(i, 1)}+B_{i}\right)+\sum_{\mathcal{I}_{j}} \frac{1}{2}\left(c_{j(i, \ell+1)}-c_{j(i, \ell-1)}\right)\right) \\
\geq\left(\sum_{j=0}^{j_{1}} z_{j}\right)-\left(\sum_{j=0}^{j_{1}} z_{j}\right)=0 .
\end{gathered}
$$


We have thus dealt with the first index if it falls inside the Riemann-Roch region. We may repeat the argument at each $j_{e}$ in the Riemann-Roch region successively, stopping when either the $j_{e}$ 's are exhausted or we reach the Clifford region. Continuing on, if $j_{e}=j_{R R}$ so that $j_{e}+1=j_{C l i f f}$, or if $j_{e}$ and $j_{e}+1$ fall within the Clifford region, then the desired results follow by similar arguments using the Clifford bounds (6) in place of the Riemann-Roch bounds (5).

Ideally, we would now show that the bound obtained in Lemma 6.1 is smaller than what is required in the numerical criterion. Unfortunately, this is not always true. Lemma 6.1 is sufficient for many, but not all, sets of linearizing weights $\mathcal{B}$. Below we have listed five cases which exhaust all possibilities. In Cases A-C, we can prove asymptotic stability of smooth curves. In Cases D and E, we cannot prove stability, so we will ultimately impose hypotheses to ensure that these cannot occur.

Choose any sufficiently small value $\epsilon>0$. (The role of $\epsilon$ will become clear in the proof of Theorem 7.1.) Then we consider the following five cases:

$$
\begin{array}{ll}
\text { Case A. } & n \geq 1 \text { and } b \geq \frac{g-1}{N}+\epsilon(N+1), \\
\text { Case B. } & n \geq 1 \text { and } b<\frac{g-1}{N}+\epsilon(N+1)<\frac{1}{2}, \\
\text { Case C. } & n=0 \text { and } N \geq 2 g-2, \\
\text { Case D. } & n=0 \text { and } N<2 g-2, \\
\text { Case E. } & n \geq 1 \text { and } b<\frac{g-1}{N}+\epsilon(N+1) \geq \frac{1}{2} .
\end{array}
$$

Consider Case A: To apply Lemma 6.1 to our problem, we need to bound $\sum_{j=0}^{\bar{N}} Z_{j} r_{j}$. Let $r_{N-g+1}, \ldots, r_{N-1}, r_{N}=0$ be the last $g$ weights (that is, ignore the index $j$ and list the smallest weights as many times as indicated by their multiplicities). Then we have

$$
\sum_{j=0}^{\bar{N}} Z_{j} r_{j} \leq \sum z_{j} r_{j}+r_{N-g+1}+\cdots+r_{N} \leq 1+r_{N-g+1}+\cdots+r_{N}
$$

The next lemma gives a bound for $r_{N-g+1}+\cdots+r_{N}$.

Lemma 6.2. $r_{N-g+1}+\cdots+r_{N} \leq \frac{g-1}{N}$.

Proof. Recall that $r_{N}=0$, so we may omit it from all the following sums. We argue similarly to Morr, Theorem 4.1. We wish to maximize $r_{N-g+1}+\cdots+r_{N-1}$, which is linear in the $r$ 's, subject to the constraints $\sum_{j=0}^{\bar{N}-1} z_{j} r_{j}=1$ and that the $r$ 's are decreasing. In the affine hyperplane in an $(N-1)$-dimensional $r$-space determined by the equation $\sum_{j=0}^{\bar{N}-1} z_{j} r_{j}=1$, the condition that the $r$ 's are decreasing defines an $(N-1)$-simplex. The vertices of this simplex correspond to sequences of the following form:

$$
r_{0}=\cdots=r_{h}>r_{h+1}=\cdots=r_{N-1}=0 .
$$

The function must take its maximum at (at least) one of these vertices, and it is easy to check that the maximum occurs when

$$
r_{0}=\cdots=r_{N-1}>0,
$$

or $r_{j}=\frac{1}{N}$ for all $j$, yielding a maximum value of $\frac{g-1}{N}$. 
Also, the defining hypothesis of Case A in (45) may be written as follows:

$$
\begin{aligned}
b & \geq \frac{g-1}{N}+\epsilon(N+1) \\
\Leftrightarrow \frac{g-1}{N} & \leq \frac{g-1+b}{N+1}-\epsilon .
\end{aligned}
$$

Therefore, as a trivial extension of Lemma 6.2, we have:

$$
r_{N-g+1}+\cdots+r_{N} \leq \frac{g-1+b}{N+1}-\epsilon .
$$

We combine (46) with the bound found in (43) to obtain:

$$
T^{\mathrm{vir}} \leq\left(1+\frac{g-1+b}{N+1}-\epsilon\right) u^{2} v^{2}+\left(\sum_{i=1}^{q} B_{i} r_{j(i, 0)}\right)\left(2 u v^{2}+v^{2}\right)+(d u v+d v-g+1) v r_{0} .
$$

Note that the leading coefficient $1+\frac{g-1+b}{N+1}-\epsilon$ is less than the leading coefficient $1+\frac{g-1+b}{N+1}$ of the numerical criterion (2) by $\epsilon$. This completes our discussion of Case A.

Next consider Cases B and C, defined in (45). In these cases, the bound given in Lemma 6.2 is too large to use with the numerical criterion. Fortunately, if we examine the proof of Lemma 6.1 closely, we can improve the bound there a little bit and arrive at the same result (47) as in Case A, provided that $\epsilon$ is sufficiently small. (The calculation easily yields precise bounds for $\epsilon$.)

Unfortunately, in Cases D and E, we know of no way to improve the bound of Lemma 6.1 in order to get the leading coefficient of $T^{\text {vir }}$ small enough to use with the numerical criterion in this case. Therefore, in the GIT-Stability Theorem of the next section, we will include hypotheses to ensure that only Cases A, B, C may occur.

\section{GIT-Stability of SMooth POINTED CURVES}

7.1. The GIT-Stability Theorem. We are ready to prove the main result of this paper:

Theorem 7.1 (GIT-Stability Theorem). Suppose $g, n, d, \mathcal{B}$, and $\epsilon$ are given, and that they satisfy one of the following three sets of hypotheses:

A. $\quad n \geq 1, \quad b \geq \frac{g-1}{N}+\epsilon(N+1)$.

B. $\quad n \geq 1, \quad b<\frac{g-1}{N}+\epsilon(N+1)<\frac{1}{2}, \quad \epsilon \leq \frac{1}{2 N(N+1)}(N-2 g+3-2 b)$.

C. $n=0, \quad N \geq 2 g-2, \quad \epsilon \leq \frac{1}{2 N(N+1)}(N-2 g+3)$.

Consider a point in the incidence locus I parametrizing a genus $g$ smooth pointed curve $\left(C \subset \mathbf{P}^{N}, P_{1}, \ldots, P_{n}\right)$ embedded in $\mathbf{P}^{N}$ by a complete linear system of degree $d$. (We do not assume that the points $P_{i}$ are distinct, nor that $C$ is pluricanonically embedded.)

If $n \geq 1$, suppose that $\mathcal{B}$ satisfies the following condition: for any subset $I \subseteq$ $[1 \ldots n]$ for which the marked points $P_{i}$ coincide, $\sum_{i \in I} b_{i}<\frac{1}{2}$. In particular, applying this to the singletons $I=\{i\}$, we have that $b_{i}<1 / 2$ for $i=1, \ldots, n$.

Let $m=(u+1) v$. Then for certain large values of $m$, the point of I parametrizing $\left(C \subset \mathbf{P}^{N}, P_{1}, \ldots, P_{n}\right)$ is GIT-stable for the $S L(N+1)$-action with the linearization 
specified by $m_{i}^{\prime}=b_{i} m^{2}$ for each $i$. More precisely, there exist:

(1) a positive integer $u_{0}$ depending on $g, n, d, \mathcal{B}$ and $\epsilon$, but not on the curve $C$, the points $P_{i}$, or the embedding $C \subset \mathbf{P}^{N}$; and

(2) a function $v_{0}(u)$ whose domain is all integers greater than $u_{0}$, and which depends on $g, n, d, \mathcal{B}, \epsilon$ and $u$, but not on the curve $C$, the points $P_{i}$, or the embedding $C \subset \mathbf{P}^{N}$

such that for any integers $u \geq u_{0}$ and $v \geq v_{0}(u)$, the point of I parametrizing $\left(C \subset \mathbf{P}^{N}, P_{1}, \ldots, P_{n}\right)$ is GIT-stable for the $S L(N+1)$-action with the linearization specified by $m_{i}^{\prime}=b_{i} m^{2}$ for each $i$.

Proof. By the numerical criterion, it is enough to show $\lambda$-stability for an arbitrary 1-PS $\lambda$ of $S L(N+1)$. Suppose $\lambda$ is given. We apply all the preceding constructions to $\lambda$. In particular, associated to $\lambda$ we have the filtration $\tilde{X}_{\bullet}$ and its profile and virtual profile.

By (47) and (35) we have

$$
\begin{aligned}
T= & T^{\mathrm{vir}}+\Delta \\
\leq & \left(1+\frac{g-1+b}{N+1}-\epsilon\right) u^{2} v^{2}+\left(\sum_{i=1}^{q} b_{i} r_{j(i, 0)}\right)\left(2 u v^{2}+v^{2}\right) \\
& +(d u v+d v-g+1) v r_{0}+\frac{9}{2} d u v^{2}+\frac{9}{2} d v^{2} \\
\leq & \left(1+\frac{g-1+b}{N+1}-\epsilon\right) u^{2} v^{2}+\left(2 \sum_{i=1}^{n} b_{i} r_{j(i, 0)}+\frac{11}{2} d\right) u v^{2} \\
& +\left(2 \sum_{i=1}^{n} b_{i} r_{j(i, 0)}+\frac{11}{2} d\right) v^{2}+v \\
\leq & \left(1+\frac{g-1+b}{N+1}-\epsilon\right) u^{2} v^{2}+\left(2 b+\frac{11}{2} d\right) u v^{2}+\left(2 b+\frac{11}{2} d\right) v^{2}+v .
\end{aligned}
$$

Recall the bound required in the numerical criterion:

$$
\begin{aligned}
(1+ & \left.\frac{g-1+b}{N+1}\right) m^{2}-\frac{g-1}{N+1} m=\left(1+\frac{g-1+b}{N+1}\right)\left(u^{2} v^{2}+2 u v^{2}+v^{2}\right) \\
& -\frac{g-1}{N+1}(u v+v) .
\end{aligned}
$$

We want to show that the RHS of (48) is less than (49), or equivalently that

$$
\begin{gathered}
0 \leq\left(\left(\frac{g-1+b}{N+1}-\left(\frac{g-1+b}{N+1}-\epsilon\right)\right) u^{2}+\left(2+\frac{2 g-2+2 b}{N+1}-2 b-\frac{11}{2} d\right) u\right. \\
\left.+\left(1+\frac{g-1+b}{N+1}-2 b-\frac{11}{2} d\right)\right) v^{2}-\left(\frac{g-1}{N+1}(u+1)+1\right) v .
\end{gathered}
$$

But the coefficient of $u^{2}$ in the coefficient of $v^{2}$ is $\epsilon>0$. So there exists a $u_{0}$ such that for all $u \geq u_{0}$, the polynomial

$$
\epsilon u^{2}+\left(2+\frac{2 g-2+2 b}{N+1}-2 b-\frac{11}{2} d\right) u+\left(1+\frac{g-1+b}{N+1}-2 b-\frac{11}{2} d\right)
$$


is positive. Also, for all sufficiently large $v$ (that is, for all $v \geq v_{0}(u)$ ), the polynomial

$$
\begin{array}{r}
\left(\epsilon u^{2}+\left(2+\frac{2 g-2+2 b}{N+1}-2 b-\frac{11}{2} d\right) u+\left(1+\frac{g-1+b}{N+1}-2 b-\frac{11}{2} d\right)\right) v^{2} \\
-\left(\frac{g-1}{N+1}(u+1)+1\right) v
\end{array}
$$

is positive, too. Once again, we emphasize that the size of $u$ required depends on $g, n, d, \mathcal{B}$, and $\epsilon$, but not on the particular curve $C$, the points $P_{i}$, the embedding $C \subset \mathbf{P}^{N}$, or the 1-PS $\lambda$. Similarly the size of $v$ required depends on $g, n, d, \mathcal{B}, \epsilon$, and $u$, but not on $\left(C \subset \mathbf{P}^{N}, P_{1}, \ldots, P_{n}\right)$ or $\lambda$.

\section{Constructing moduli spaces}

Our motivation for studying this stability problem was to give GIT constructions of moduli spaces of DM-stable weighted pointed curves. We now discuss this application.

Let $\left(C, P_{1}, \ldots, P_{n}, \mathcal{A}\right)$ be a DM-stable weighted pointed curve with $n$ marked points. Write $a:=\sum a_{i}$ and assume that $2 g-2+a>0$. Then for $\nu$ sufficiently large and divisible, $\left(\omega_{C}\left(\sum a_{i} P_{i}\right)\right)^{\otimes \nu}=: \mathcal{O}_{C}(1)$ is a very ample line bundle. Write

$$
\begin{aligned}
d & =\operatorname{deg}\left(\omega_{C}\left(\sum a_{i} P_{i}\right)\right)^{\otimes \nu}=\nu(2 g-2+a), \\
N+1 & =\operatorname{dim} H^{0}\left(C,\left(\omega_{C}\left(\sum a_{i} P_{i}\right)\right)^{\otimes \nu}\right)=\nu(2 g-2+a)-g+1, \\
P(t) & =h^{0}\left(C, \mathcal{O}_{C}(t)\right)=d t-g+1 .
\end{aligned}
$$

Write $I \subset \operatorname{Hilb}\left(\mathbf{P}^{N}, P(t)\right) \times \prod_{i=1}^{n} \mathbf{P}^{N}$ for the incidence locus where the points in the second factor land on the curve $C \subset \mathbf{P}^{N}$ parametrized by a point of the first factor. Write $J$ for the locally closed subscheme of $I$ parametrizing weighted pointed curves embedded by $\left(\omega_{C}\left(\sum a_{i} P_{i}\right)\right)^{\otimes \nu}$. Then the DM-stable weighted pointed curve $\left(C, P_{1}, \ldots, P_{n}, \mathcal{A}\right)$ is represented by a $P G L(N+1)$-orbit in the locus $J$.

See Section 1.1 for a description of the linearizations on $I$ under consideration.

It is important to remember that $d, N, P(t)$ all depend on $g, n, \mathcal{A}$ and $\nu$. Hence, $I$ and $J$ also depend on these parameters as well. So, even if $g$ and $n$ are held constant, if $\mathcal{A}$ or $\nu$ varies, one is moving between loci in different Hilbert schemesthat is, one is using different parameter spaces - and this is not a variation of GIT in the sense of Thaddeus and Dolgachev and Hu. On the other hand, if $g, n, \mathcal{A}$, and $\nu$ are held constant and only $m$ and $\mathcal{B}$ are allowed to vary, this is VGIT in the sense of Thaddeus and Dolgachev and $\mathrm{Hu}$.

The following theorem is the main application of the GIT-stability result of this paper:

Theorem 8.1 (Construction Theorem). Given g, $n$, and a set of embedding weights $\mathcal{A}$ with $0 \leq a_{i}<1$ for $i=1, \ldots, n$, and satisfying $2 g-2+a>0$, it is possible to choose $\nu, m$, and a set of linearizing weights $\mathcal{B}$ such that the GIT quotient $J / / m, \mathcal{B} S L(N+1) \cong \bar{M}_{g, \mathcal{A}}$. In particular, for $1 / 2+\epsilon<a_{i}<1$, this gives GIT constructions of $\bar{M}_{g, n}$, since for such weights $\bar{M}_{g, \mathcal{A}} \cong \bar{M}_{g, n}$.

A full proof of Theorem 8.1 may be found in Swin, Ch. 3 and App. A. The novel part for weighted pointed DM-stable curves is the GIT-Stability Theorem, Theorem 7.1 . 
The construction follows a strategy established by Gieseker and Mumford which may now be considered standard (see also [HM], Ch. 4 and [BS]). First, by the GIT-Stability Theorem, Theorem 7.1, we know that for certain embeddings and linearizations, smooth pointed curves are GIT-stable. (The analogous GIT-stability proof in $\mathrm{BS}$, is the only part of the construction where $[\mathrm{BS}$ ] cannot handle adding weights $\mathcal{A}$ to the marked points. The argument there will not work if an elliptic tail is glued to a marked point of weight $<1$.)

Next, by careful examination of the GIT-stable locus, one concludes that for certain embedding degrees $\nu$ and linearizations, anything GIT-stable must be a reduced nodal curve, and marked points must be nonsingular if $a_{i}>0$ and may only collide if $\sum_{i \in I} a_{i} \leq 1$. Harris and Morrison call results of this type "Potential Stability" theorems. One shows successively that the curve $C \subset \mathbf{P}^{N}$ is nondegenerately embedded, is generically reduced, has no singularities of multiplicity $\geq 2$, and has no cusps or tacnodes. For each of these results, it is generally easy to write a 1-PS which is adapted to the geometry of the situation at hand and to show that this 1-PS is destabilizing. For example, to prove that cuspidal curves are GIT-unstable, one looks at a 1-PS which has negative weight on sections vanishing at the cusp. We do not write a full proof of the Potential Stability Theorem here, as nearly all the analogous arguments in BS] go through unchanged when weights $\mathcal{A}$ are added to the picture. Indeed, Sections 5.1-5.3 and 5.5 of [BS go through unchanged.

One obvious change is needed from $\mathrm{BS}$, since marked points behave differently on $\bar{M}_{g, n}$ and $\bar{M}_{g, \mathcal{A}}$. It is a straightforward exercise to adapt the results of [BS], Section 5.4 to the case of weighted marked points. In doing so, one is naturally led to use a linearization of the form $b_{i} \approx a_{i} / 2$.

Finally, a deformation argument smoothing the curve is used to prove a nodal DM-stable weighted pointed curve is GIT-stable. One must prove that the locus $J$ is closed in the GIT-semistable locus. Here again, the argument of [BS], Theorem 5.21 goes through unchanged when weights are added to the marked points.

\section{ACKNOWLEDGEMENTS}

It is with great pleasure that the author thanks his advisors, Ian Morrison and Michael Thaddeus, for their help with this work, which was the core of his 2008 Columbia University Ph.D. dissertation. The author is also very grateful to Frances Kirwan, who first got him interested in GIT and moduli spaces, and to Elizabeth Baldwin for sharing much of her early work. Finally, the author would like to thank Johan de Jong and Brendan Hassett for their technical help and encouragement.

\section{REFERENCES}

[BS] Baldwin, E. and D. Swinarski. "A geometric invariant theory construction of moduli spaces of stable maps." Int. Math. Res. Papers, 2008 no. 1, article ID rpn004, 104 pages. MR2431236 (2009f:14018)

[DH] Dolgachev, I. and Y. Hu. "Variation of geometric invariant theory quotients." Publ. Math. I.H.E.S. 87 (1998), 5-56. MR.1659282 (2000b:14060)

[Gies] Gieseker, D. Lectures on Moduli of Curves. Tata Institute Lecture Notes, Springer, 1982. MR691308 (84h:14035)

[G2] Gieseker, D. "Geometric invariant theory and applications to moduli problems." 45-73, LNM 996, Springer, 1983. MR718126 (85b:14014)

[HM] Harris, J. and I. Morrison. Moduli of Curves. Graduate Texts in Mathematics 107, Springer, 1998. MR:1631825 (99g:14031) 
[Hass] Hassett, B. "Moduli spaces of weighted pointed stable curves." Adv. Math. 173, no. 2 (2003), 316-352. MR 1957831 (2004b:14040)

[HH] Hassett, B. and D. Hyeon. "Log canonical models for the moduli space of curves: First divisorial contraction." Trans. AMS 361 (2009), 4471-4489. MR.2500894 (2009m:14039)

[Morr] Morrison, I. "Projective Stability of Ruled Surfaces." Inv. Math. 56 (1980), 269-304. MR.561975 (81c:14007)

[GIT] Mumford, D., Fogarty, J. and F.C. Kirwan. Geometric Invariant Theory. Third Edition. Springer, 1994. MR719371 (86a:14006)

[Mum3] Mumford, D. "Stability of Projective Varieties." Enseignement Math. (2) 23 (1977), no. 1-2, 39-110. MR0450272(56:8568)

[Swin] Swinarski, D. "Geometric invariant theory and moduli spaces of pointed curves." Ph.D dissertation, Columbia University, 2008. MR2626882

[Thad] Thaddeus, M. "Geometric invariant theory and flips." J. Amer. Math. Soc. 9 (1996), no. 3, 691-723. MR1333296 (96m:14017)

Department of Mathematics, University of Georgia, Athens, Georgia 30606

E-mail address: davids@math.uga.edu

Current address: Department of Mathematics, Fordham University, New York, New York 10023

E-mail address: dswinarski@fordham.edu 